\title{
Statistics of Distinguishable Particles and Resolution of the Gibbs Paradox of the First Kind
}

\author{
Hjalmar Peters
}

Received: 20 February 2010 / Accepted: 11 October 2010 / Published online: 27 October 2010

(C) The Author(s) 2010. This article is published with open access at Springerlink.com

\begin{abstract}
In physics, there are two distinct paradoxes, which are both known as the "Gibbs paradox". This article is concerned with only one of them: the false increase in entropy, which is calculated from the process of combining two gases of the same kind consisting of distinguishable particles. In the following, this paradox will be referred to as the Gibbs paradox of the first kind (GP1). (Two particles are said to be distinguishable if they are either non-identical, that is, if they have different properties, or if they are identical and there are microstates which change under transposition of the two particles.) The GP1 is demonstrated and subsequently analyzed. The analysis shows that, for (quantum or classical) systems of distinguishable particles, it is generally uncertain of which particles they consist. The neglect of this uncertainty is the root of the GP1. For the statistical description of a system of distinguishable particles, an underlying set of particles, containing all particles that in principle qualify for being part of the system, is assumed to be known. Of which elements of this underlying particle set the system is composed, differs from microstate to microstate. Thus, the system is described by an ensemble of possible particle compositions. The uncertainty about the particle composition contributes to the entropy of the system. Systems for which all possible particle compositions are equiprobable will be called harmonic. Classical systems of distinguishable identical particles are harmonic as a matter of principle; quantum or classical systems of non-identical particles are not necessarily harmonic, since for them the composition probabilities depend individually on the preparation of the system. Harmonic systems with the same underlying particle set are always correlated; hence, for harmonic systems, the entropy is no longer additive and loses its thermodynamic meaning. A quantity derived from entropy is introduced, the reduced entropy, which, for harmonic systems, replaces the entropy as thermodynamic potential. For identical classical particles, the equivalence (in particular with respect to the second law of thermodynamics) between distinguishability and indistinguishability is proved. The resolution of the GP1 is demonstrated applying the previously found results.
\end{abstract}

H. Peters (凶)

Karlsruhe Institute of Technology, Karlsruhe, Germany

e-mail: unou@rz.uni-karlsruhe.de

H. Peters $(\varangle)$

e-mail: hjalmar.peters@web.de 
Keywords Entropy · Distinguishable particles · Gibbs paradox

\section{Introduction and Outline}

In physics, there are two distinct paradoxes, which are both known as the "Gibbs paradox" $[2,3,20]$ and are often confused with each other. In the following, the false increase in entropy, which is calculated from the process of combining two gases of the same kind consisting of distinguishable particles, will be referred to as the Gibbs paradox of the first kind (GP1) [16, 21-23, 30, 38, 41, 44]. The Gibbs paradox of the second kind (GP2) addresses the fact that the entropy increase, when combining two gases of different kinds, is independent of the degree of similarity between the two kinds of gases, and that this entropy increase vanishes discontinuously at the transition from similarity to sameness $[4,7,18,24,25,46]$. The GP2 is not the subject of the present paper.

Classical statistical mechanics yields, for an ideal gas of pairwise distinguishable particles, the entropy (3.4), which is not extensive and therefore leads to the GP1 (see Sect. 3.1.1). For an ideal gas of indistinguishable particles, however, one obtains the extensive entropy (3.7), and so the GP1 does not emerge in this case. Schrödinger [38], Landé [23] and others (e.g., Huang [21]) regarded classical particles as inherently distinguishable; thus, to them, the GP1 posed a fundamental inconsistency of classical statistical mechanics. Gibbs [16], Tetrode [43] and others (particularly explicitly, Hestenes [19]), on the other hand, inferred from the GP1 that classical particles of the same kind are indistinguishable (in Gibbs's terminology: one has to use "generic phases"). In the opinion of the present author, neither position is correct: in Sect. 5.6 it is shown that distinguishable and indistinguishable classical particles of the same kind are physically equivalent, and in Sect. 6.1.2 the resolution of the GP1 for distinguishable classical particles of the same kind is presented.

The key to the resolution of the GP1 and the central idea of this article is the insight that, for a system of distinguishable particles, there generally is no certainty of which particles the system consists, that is, the system must be described by an ensemble of possible particle compositions. The uncertainty about the particle composition contributes to the entropy of the system (see Sect. 4.1) and entails that the system is correlated with certain other systems (see Sects. 4.3.2 and 5.4). The consideration of this uncertainty in the statistical treatment of systems of distinguishable particles leads to a new statistics, described in Sects. 4 and 5. Within the framework of this statistics, the resolution of the GP1 is evident (Sect. 6).

In Sect. 2, the basic terms used in this article are introduced. In doing so, quantum and classical mechanics are treated in parallel. At first, in Sect. 2.1.1, the state space of a quantum system is defined as the direct sum of conventional many-particle state spaces. This allows the system to consist of different particles in different microstates. Similarly, classical microstates are represented by points, which may lie in different phase spaces (Sect. 2.1.2). In Sect. 2.2, the transposition of two particles, in particular, the transposition of a system particle with a particle not belonging to the system, is defined. Two particles are called identical if all their state-independent properties agree; if in addition the transposition of the two particles leaves every microstate unchanged, then the particles are called indistinguishable (Sect. 2.5). While identical quantum particles are indistinguishable, identical classical particles can be assumed to be either distinguishable or indistinguishable (Sects. 2.6 and 2.7).

In Sect. 3, the GP1 is treated. At first, in Sect. 3.1.1, the GP1 is demonstrated for pairwise non-identical (quantum or classical) particles: in an ideal gas of pairwise non-identical particles in equilibrium, a partition is first inserted and then removed; the removal of the partition paradoxically seems to increase the entropy of the gas. This demonstration shows 
that the GP1, contrary to popular belief, is by no means only a problem of classical statistical mechanics, but may very well occur for quantum systems, too. (The interpretation of the GP1 as a pointer away from classical mechanics towards quantum mechanics [23, 38] would therefore even then be untenable, if the GP1 were insoluble.) The GP1 also arises for distinguishable identical (classical) particles (Sect. 3.1.2). Indistinguishable particles do not suffer from the GP1 (Sect. 3.1.3). In Sect. 3.2, the reason for the seeming entropy decrease during the partitioning of a system of pairwise non-identical particles is examined. As it turns out, the reason is that, in the calculation of the entropy of the partitioned system, the uncertainty about which particles are located in which subsystem is ignored; if this uncertainty is taken into consideration, then the same entropy is obtained for the system after the partitioning as before the partitioning. Thus, the GP1 leads to the insight that the particle composition of a system of non-identical (more generally, distinguishable) particles is usually not determined with certainty, that is, such a system must be described by an ensemble of possible particle compositions.

Section 4 is concerned with systems of pairwise non-identical particles. At first, a closed system $\mathbb{S}$ of $N$ particles is regarded. Of which $N$ particles $\mathbb{S}$ consists, may vary from microstate to microstate; only an underlying set $\mathcal{P}$ of $\mathfrak{N} \geq N$ pairwise non-identical particles, containing all particles that in principle qualify for being part of $\mathbb{S}$, is known. Part of the entropy of $\mathbb{S}$ can directly be ascribed to the uncertainty about the particle composition of $\mathbb{S}$ (Sect. 4.1). $\mathbb{S}$ will be called $\mathcal{P}$-harmonic if all its $\left(\begin{array}{l}\mathfrak{N} \\ N\end{array}\right)$ possible particle compositions are equiprobable. Because particles cannot be located in one system with certainty while at the same time being located in another system with certainty, harmonic systems with the same underlying particle set are always correlated. Hence, the entropy of a total system, consisting of two harmonic subsystems, is not additively composed of the entropies of the two subsystems (Sect. 4.3.2). With the loss of additivity, the entropy also loses its thermodynamic meaning for harmonic systems: for harmonic systems, no longer the entropy, but the reduced entropy, defined in Sect. 4.3.3.1, is a thermodynamic potential (Sect. 4.3.3.2).

In Sect. 5, systems of pairwise distinguishable identical classical particles are discussed. At first, a closed system $\mathbb{S}$ of $N$ identical particles is regarded. $\mathcal{R}$ is the set of all particles that are identical to the particles in $\mathbb{S}$. Since microstates that arise from each other through permutation of $\mathcal{R}$-particles are experimentally indiscernible, it is postulated in Sect. 5.1 that they are equiprobable. From this postulate immediately follows the $\mathcal{R}$-harmonicity of systems of $\mathcal{R}$-particles (Sect. 5.2); thus, those results of Sect. 4.3 that were deduced in particular for harmonic systems of non-identical particles hold for systems of distinguishable identical classical particles in general (Sects. 5.4 and 5.5). In Sect. 5.6, it is shown that distinguishable and indistinguishable identical classical particles are physically equivalent, that is, equivalent both mechanically (Sect. 5.6.5) and regarding the second law of thermodynamics (Sect. 5.6.6.1).

Section 6 finally turns towards the GP1 again. Now, with the results of Sects. 4 and 5 , it can easily be shown that there is no change in entropy when combining ideal gases of the same kind, consisting of distinguishable identical classical particles (Sect. 6.1.2) or of non-identical particles (Sect. 6.2.2): the entropy of systems of distinguishable particles is neither extensive nor additive; in the calculation of the entropy change caused by the process of combining, the contributions that originate from the non-extensivity and from the non-additivity mutually compensate each other.

In Sect. 7, at first, the essential results are once again summarized. After that, the relevance of the results, presented in this article, is discussed. 


\section{States and Particles}

\subsection{States}

\subsubsection{Quantum Mechanics}

The pure (quantum) states of a physical system are represented by vectors of the state space $\mathcal{H}$ (a Hilbert space) of the system; if $s$ is a pure state, then $|s\rangle \in \mathcal{H}$ denotes a vector representing s. Those vectors of $\mathcal{H}$ that represent pure states are called state vectors. State vectors are normalized and satisfy the symmetrization postulate (see Sect. 2.6.1). Two state vectors represent the same pure state if and only if they arise from each other through multiplication with a phase factor (i.e., a complex scalar of absolute value 1).

In this article, the state space is the direct sum of vector subspaces:

$$
\mathcal{H}=\bigoplus_{i=1}^{\mathrm{N}} \mathcal{U}_{i}
$$

Each vector subspace $\mathcal{U}_{i}$, in turn, is the many-particle state space (i.e., the tensor product of the single-particle state spaces) of $N_{i}$ particles $\mathfrak{p}_{n_{i, 1}}, \mathfrak{p}_{n_{i, 2}}, \ldots, \mathfrak{p}_{n_{i, N_{i}}}\left(n_{i, 1}, n_{i, 2}, \ldots, n_{i, N_{i}} \in \mathbb{N}\right.$; $\mathfrak{p}_{1}, \mathfrak{p}_{2}, \ldots$ are particle labels):

$$
\mathcal{U}_{i}=\bigotimes_{j=1}^{N_{i}} \mathcal{V}_{n_{i, j}}
$$

$\left(\mathcal{V}_{n_{i, j}}\right.$ denotes the single-particle state space of the particle $\left.\mathfrak{p}_{n_{i, j}}\right)$. The state vectors of $\mathcal{U}_{i}$ represent the pure states in which the system is composed of the particles $\mathfrak{p}_{n_{i, 1}}, \mathfrak{p}_{n_{i, 2}}, \ldots, \mathfrak{p}_{n_{i, N_{i}}}$ (referred to as $\mathcal{U}_{i}$-particles in the following). In every pure state, the system has a welldefined particle composition (and therefore also a well-defined particle number), that is, every state vector lies in one of the vector subspaces $\mathcal{U}_{1}, \mathcal{U}_{2}, \ldots, \mathcal{U}_{\mathrm{N}}$. Conversely, all normalized vectors of the vector subspaces $\mathcal{U}_{1}, \mathcal{U}_{2}, \ldots, \mathcal{U}_{\mathrm{N}}$ that satisfy the symmetrization postulate are state vectors. Those particles of which the system is composed in a pure state $s$ (and no other particles) are system particles in $\mathrm{s}$.

The macrostate $\mathrm{M}$ of a quantum system is represented by exactly one density operator $\rho$ acting upon $\mathcal{H}$. Density operators are positive-semidefinite Hermitian operators of trace 1; thus, in particular, there is an orthonormal basis $\mathcal{B}$ of $\mathcal{H}$ consisting of eigenvectors of $\rho . \rho$ is restricted by the fact that it must be possible to choose $\mathcal{B}$ such that each $\mathcal{B}$-vector lies in one of the vector subspaces $\mathcal{U}_{1}, \mathcal{U}_{2}, \ldots, \mathcal{U}_{\mathrm{N}}$. (In the following, such a choice is assumed.) The pure states represented by those $\mathcal{B}$-vectors that are state vectors (i.e., those $\mathcal{B}$-vectors that satisfy the symmetrization postulate) are called microstates (of M). Normally, the set $\mathcal{M}$ of microstates of $\mathrm{M}$ is not unambiguously determined, since there is some leeway in the choice of $\mathcal{B}$. If the $\mathcal{B}$-vector $|\mathrm{m}\rangle$ representing the microstate $\mathrm{m} \in \mathcal{M}$ is an eigenvector of $\rho$ with the eigenvalue $P$, then $P$ is the probability of $\mathrm{m}$ in $\mathrm{M}$. $\mathcal{B}$-vectors representing no microstates have to correspond to the eigenvalue zero.

From the orthonormality of $\mathcal{B}$ it immediately follows that

$$
\langle\mathrm{m} \mid \mathrm{n}\rangle=\delta_{\mathrm{m}, \mathrm{n}} \quad \forall \mathrm{m}, \mathrm{n} \in \mathcal{M}
$$

$\left(\delta_{m, n}\right.$ is the Kronecker delta and $\langle\mathrm{m} \mid \mathrm{n}\rangle$ is the inner product of the vectors $|\mathrm{m}\rangle$ and $|\mathrm{n}\rangle$ in Dirac notation). Since $\rho$ is positive-semidefinite, the probability $P_{\mathrm{M}}(\mathrm{m})$ of $\mathrm{m} \in \mathcal{M}$ in $\mathrm{M}$ satisfies 
the condition

$$
P_{\mathrm{M}}(\mathrm{m}) \geq 0 \quad \forall \mathrm{m} \in \mathcal{M} .
$$

Furthermore, the normalization condition

$$
\sum_{\mathrm{m} \in \mathcal{M}} P_{\mathrm{M}}(\mathrm{m})=1
$$

is satisfied because the trace of $\rho$ is 1 . Finally, the spectral decomposition

$$
\rho=\sum_{\mathrm{m} \in \mathcal{M}} P_{\mathrm{M}}(\mathrm{m})|\mathrm{m}\rangle\langle\mathrm{m}|
$$

shows that $M$ is unambiguously determined by its microstates and their probabilities.

The entropy $S$ of $\mathrm{M}$ is defined as

$$
S(\mathrm{M}):=-k \sum_{\mathrm{m} \in \mathcal{M}} P_{\mathrm{M}}(\mathrm{m}) \ln P_{\mathrm{M}}(\mathrm{m})
$$

where $k$ is Boltzmann's constant. Here and in the following, $0 \ln 0$ is set to zero. $S(\mathrm{M})$ is independent of the choice of the microstates and thus is well-defined [8].

Remark 2.1 The system generally is not composed of the same particles in all microstates possessing a non-vanishing probability in M. Hence, in general, the particle composition (and the particle number) of the system in $\mathrm{M}$ is undetermined, that is, $\mathrm{M}$ describes an ensemble of possible particle compositions.

\subsubsection{Classical Mechanics}

Every (classical) microstate of a physical system is represented by exactly one point in one of the phase spaces $\mathcal{U}_{1}, \mathcal{U}_{2}, \ldots, \mathcal{U}_{\mathrm{N}}$ of the system. Conversely, each phase space point in

$$
\mathcal{H}:=\bigcup_{i=1}^{\mathrm{N}} \mathcal{U}_{i}
$$

represents a microstate. If $\mathrm{m}$ is a microstate, then $\widehat{\mathrm{m}} \in \mathcal{H}$ denotes the phase space point representing $\mathrm{m}$.

Each phase space $\mathcal{U}_{i}$ is spanned by the generalized coordinates and the conjugate generalized momenta of certain particles, the $\mathcal{U}_{i}$-particles. The points in $\mathcal{U}_{i}$ represent the microstates in which the system is composed of the $\mathcal{U}_{i}$-particles. The $\mathcal{U}_{i}$-particles (and no others) are system particles in those microstates. In the following, $\zeta_{i}$ denotes the set of $\mathcal{U}_{i}$-particles and $\left\langle\zeta_{i} ; \vec{q}, \vec{p}\right\rangle$ the point in $\mathcal{U}_{i}$ with the phase space coordinates (i.e., the generalized coordinates and momenta of the $\mathcal{U}_{i}$-particles) $\vec{q}, \vec{p}$.

The macrostate $\mathrm{M}$ of a classical system is represented by a density function, which assigns to every phase space point $\widehat{m} \in \mathcal{H}$ a (non-negative) probability density $\rho_{\mathrm{M}}(\widehat{\mathrm{m}})$. Hence, a subset $\widehat{\mu}$ of $\mathcal{H}$ possesses in $\mathrm{M}$ the probability

$$
\int_{\widehat{\mu}} \mathrm{d} \mathcal{H} \rho_{\mathrm{M}}(\widehat{\mathrm{m}}) \stackrel{(2.8)}{:=} \sum_{i=1}^{\mathrm{N}} \int_{\widehat{\mu} \cap \mathcal{U}_{i}} \mathrm{~d} \mathcal{U}_{i} \rho_{\mathrm{M}}(\widehat{\mathrm{m}})
$$




$$
=\sum_{i=1}^{\mathrm{N}} \int_{\left\{(\vec{q}, \vec{p}):\left\langle\zeta_{i} ; \vec{q}, \vec{p}\right\rangle \in \widehat{\mu}\right\}} \mathrm{d}^{\frac{\operatorname{dim} \mathcal{U}_{i}}{2}} q \mathrm{~d}^{\frac{\operatorname{dim} \mathcal{U}_{i}}{2}} p \rho_{\mathrm{M}}\left(\left\langle\zeta_{i} ; \vec{q}, \vec{p}\right\rangle\right) .
$$

$\mathcal{H}$ itself has to possess in $\mathrm{M}$ the probability

$$
\int \mathrm{d} \mathcal{H} \rho_{\mathrm{M}}(\widehat{\mathrm{m}})=1
$$

(normalization condition). The set $\mu:=\{\mathrm{m}: \widehat{\mathrm{m}} \in \widehat{\mu}\}$ of all microstates that are represented by the phase space points in $\widehat{\mu}$ is assigned in $M$ the same probability (2.9) as $\widehat{\mu}$.

The entropy $S$ of $\mathrm{M}$ is defined as

$$
S(\mathrm{M}):=-k \sum_{i=1}^{\mathrm{N}} \int \mathrm{d} \mathcal{U}_{i} \rho_{\mathrm{M}}(\widehat{\mathrm{m}}) \ln \left[\rho_{\mathrm{M}}(\widehat{\mathrm{m}})(2 \pi \hbar)^{\frac{\mathrm{dim} \mathcal{U}_{i}}{2}}\right],
$$

assuming an elementary phase space volume of $(2 \pi \hbar)^{\frac{\operatorname{dim} \mathcal{U}_{i}}{2}}$ in the phase space $\mathcal{U}_{i}$.

Remark 2.2 The phase space points possessing a non-vanishing probability density in M generally do not all lie in the same phase space. Hence, in general, the particle composition (and the particle number) of the system in $M$ is undetermined, that is, $M$ describes an ensemble of possible particle compositions.

\subsection{Transpositions and Permutations of Particles}

\subsubsection{Transposition of Two Quantum Particles}

Let $|\Psi\rangle$ be a vector of the vector subspace $\mathcal{U}_{i} \subset \mathcal{H}$ and $\zeta_{i}:=\left\{\mathfrak{p}_{n_{i, 1}}, \mathfrak{p}_{n_{i, 2}}, \ldots, \mathfrak{p}_{n_{i, N_{i}}}\right\}$ the set of $\mathcal{U}_{i}$-particles (cf. Sect. 2.1.1). Let the single-particle state spaces of the four particles $\mathfrak{p}_{n_{i, q}}, \mathfrak{p}_{n_{i, r}} \in \zeta_{i}$ and $\mathfrak{p}_{s}, \mathfrak{p}_{t} \notin \zeta_{i}$ be isomorphic to each other such that the same basis $\left\{\left|\phi_{1}\right\rangle,\left|\phi_{2}\right\rangle, \ldots\right\}$ can be chosen for them. Let $\left\{\left|\chi_{1}\right\rangle,\left|\chi_{2}\right\rangle, \ldots\right\}$ be a basis of the many-particle state space of all $\mathcal{U}_{i}$-particles except $\mathfrak{p}_{n_{i, q}}$ and $\mathfrak{p}_{n_{i, r}}$. Because of (2.2), $|\Psi\rangle$ may be written in the following way as a linear combination of tensor product vectors (the $c_{u, v, w}$ are complexvalued coefficients):

$$
\left.|\Psi\rangle=\sum_{u, v, w} c_{u, v, w}\left|\mathfrak{p}_{n_{i, q}}: \phi_{u}\right\rangle \otimes\left|\mathfrak{p}_{n_{i, r}}: \phi_{v}\right\rangle \otimes \mid \text { all } \mathcal{U}_{i} \text {-particles except } \mathfrak{p}_{n_{i, q}} \text { and } \mathfrak{p}_{n_{i, r}}: \chi_{w}\right\rangle
$$

The transposition $\sigma_{n_{i, q}, n_{i, r}}$ of $\mathfrak{p}_{n_{i, q}}$ and $\mathfrak{p}_{n_{i, r}}$ maps $|\Psi\rangle$ to the vector

$$
\begin{aligned}
\sigma_{n_{i, q}, n_{i, r}}|\Psi\rangle:= & \sum_{u, v, w} c_{u, v, w}\left|\mathfrak{p}_{n_{i, q}}: \phi_{v}\right\rangle \otimes\left|\mathfrak{p}_{n_{i, r}}: \phi_{u}\right\rangle \\
& \left.\otimes \mid \text { all } \mathcal{U}_{i} \text {-particles except } \mathfrak{p}_{n_{i, q}} \text { and } \mathfrak{p}_{n_{i, r}}: \chi_{w}\right\rangle
\end{aligned}
$$

of $\mathcal{U}_{i}$. The transposition $\sigma_{n_{i, q}, s}$ of $\mathfrak{p}_{n_{i, q}}$ and $\mathfrak{p}_{s}$ is only then defined for $|\Psi\rangle$ if there is a vector subspace $\mathcal{U}_{j} \subset \mathcal{H}$ with the $\mathcal{U}_{j}$-particles $\mathfrak{p}_{n_{i, 1}}, \mathfrak{p}_{n_{i, 2}}, \ldots, \mathfrak{p}_{n_{i, q-1}}, \mathfrak{p}_{s}, \mathfrak{p}_{n_{i, q+1}}, \ldots, \mathfrak{p}_{n_{i, N_{i}}}$. If this is the case, then $\sigma_{n_{i, q}, s}$ maps $|\Psi\rangle$ to the vector 


$$
\begin{aligned}
\sigma_{n_{i, q}, s}|\Psi\rangle:= & \sum_{u, v, w} c_{u, v, w}\left|\mathfrak{p}_{s}: \phi_{u}\right\rangle \otimes\left|\mathfrak{p}_{n_{i, r}}: \phi_{v}\right\rangle \\
& \left.\otimes \mid \text { all } \mathcal{U}_{i} \text {-particles except } \mathfrak{p}_{n_{i, q}} \text { and } \mathfrak{p}_{n_{i, r}}: \chi_{w}\right\rangle
\end{aligned}
$$

of $\mathcal{U}_{j}$. Finally, the transposition $\sigma_{s, t}$ of $\mathfrak{p}_{s}$ and $\mathfrak{p}_{t}$ trivially leaves $|\Psi\rangle$ unchanged, that is, $\sigma_{s, t}|\Psi\rangle:=|\Psi\rangle$.

If the transposition $\sigma$ is defined for the state vector $|\mathbf{s}\rangle$, then $\sigma|\mathbf{s}\rangle$ is also a state vector and $\sigma$ maps the pure state $\mathrm{s}$ represented by $|\mathrm{s}\rangle$ to the pure state $\sigma(\mathrm{s})$ represented by $\sigma|\mathbf{s}\rangle$; thus $\sigma(\mathrm{s})$ is determined by

$$
|\sigma(\mathbf{s})\rangle=e^{\mathrm{i} \varphi} \sigma|\mathbf{s}\rangle \quad(\varphi \in \mathbb{R}),
$$

where $e^{\mathrm{i} \varphi}$ is an undetermined phase factor.

\subsubsection{Transposition of Two Classical Particles}

Let $\widehat{\mathrm{m}}$ be a point in the phase space $\mathcal{U}_{i} \subset \mathcal{H}$ and $\zeta_{i}=\left\{\mathfrak{p}_{n_{i, 1}}, \mathfrak{p}_{n_{i, 2}}, \ldots, \mathfrak{p}_{n_{i, N_{i}}}\right\}$ the set of $\mathcal{U}_{i}$ particles (cf. Sect. 2.1.2). Let the four particles $\mathfrak{p}_{n_{i, q}}, \mathfrak{p}_{n_{i, r}} \in \zeta_{i}$ and $\mathfrak{p}_{s}, \mathfrak{p}_{t} \notin \zeta_{i}$ have the same internal degrees of freedom. The transposition $\sigma_{n_{i, q}, n_{i, r}}$ of $\mathfrak{p}_{n_{i, q}}$ and $\mathfrak{p}_{n_{i, r}}$ maps $\hat{\mathrm{m}}$ to the phase space point $\sigma_{n_{i, q}, n_{i, r}}(\widehat{\mathrm{m}}) \in \mathcal{U}_{i}$. One obtains $\sigma_{n_{i, q}, n_{i, r}}(\widehat{\mathrm{m}})$ by interchanging the phase space coordinates of $\widehat{\mathrm{m}}$ associated with $\mathfrak{p}_{n_{i, q}}$ and $\mathfrak{p}_{n_{i, r}}$; that is, the coordinates of $\widehat{\mathrm{m}}$ associated with $\mathfrak{p}_{n_{i, q}}$ become the corresponding coordinates of $\sigma_{n_{i, q}, n_{i, r}}(\widehat{\mathrm{m}})$ associated with $\mathfrak{p}_{n_{i, r}}$, and vice versa. The transposition $\sigma_{n_{i, q}, s}$ of $\mathfrak{p}_{n_{i, q}}$ and $\mathfrak{p}_{s}$ is only then defined for $\widehat{\mathrm{m}}$ if there is a phase space $\mathcal{U}_{j} \subset \mathcal{H}$ with the $\mathcal{U}_{j}$-particles $\mathfrak{p}_{n_{i, 1}}, \mathfrak{p}_{n_{i, 2}}, \ldots, \mathfrak{p}_{n_{i, q-1}}, \mathfrak{p}_{s}, \mathfrak{p}_{n_{i, q+1}}, \ldots, \mathfrak{p}_{n_{i, N_{i}}}$. If this is the case, then $\sigma_{n_{i, q}, s}(\widehat{\mathrm{m}})$ lies in $\mathcal{U}_{j}$ and is determined as follows: the coordinates of $\sigma_{n_{i, q}, s}(\widehat{\mathrm{m}})$ associated with $\mathfrak{p}_{s}$ are equal to the corresponding coordinates of $\widehat{m}$ associated with $\mathfrak{p}_{n_{i, q}}$, and the coordinates of $\sigma_{n_{i, q}, s}(\widehat{\mathrm{m}})$ associated with the other $\mathcal{U}_{j}$-particles agree with the corresponding coordinates of $\widehat{\mathrm{m}}$. Finally, the transposition $\sigma_{s, t}$ of $\mathfrak{p}_{s}$ and $\mathfrak{p}_{t}$ trivially leaves $\widehat{\mathrm{m}}$ unchanged, that is, $\sigma_{s, t}(\widehat{\mathrm{m}}):=\widehat{\mathrm{m}}$.

If the transposition $\sigma$ is defined for the phase space point $\widehat{m}$, then $\sigma$ maps the microstate $\mathrm{m}$ represented by $\widehat{\mathrm{m}}$ to the microstate $\sigma(\mathrm{m})$ represented by $\sigma(\widehat{\mathrm{m}})$; thus, $\sigma(\mathrm{m})$ is determined by

$$
\widehat{\sigma(m)}=\sigma(\widehat{m})
$$

\subsubsection{Particle Permutations}

Permutations are compositions of transpositions. In the following, it is assumed that the microstates of a quantum macrostate $M$ are chosen such that, for every microstate $m$ of $M$, every particle permutation that is defined for $m$ maps $m$ to a pure state which is again a microstate of $\mathrm{M}$.

\subsection{Processes and Particle Conservation}

At any point in time, a (quantum or classical) system is in a particular macrostate. (In the following, a system is occasionally linguistically identified with the macrostate it is in; for example, the "entropy of a system" actually means the entropy of the macrostate the system is in.) The temporal evolution of the macrostate of a system will be called a process; the period during which an examined process takes place will be called the observation period. A particle is relevant to an examined process if at any point in time during the observation 
period it is a system particle with a non-vanishing probability. In the present paper, it is assumed that relevant particles can neither be created nor annihilated during the observation period. Relevant particles do not have to be elementary; however, according to the foregoing assumption, composite relevant particles (e.g., molecules composed of electrons and nuclei) must not decay or convert into other particles during the observation period.

\subsection{Particle Attributes}

Particles possess attributes such as electric charge, position and velocity. If, for each particle, the value of an attribute $A$ is independent of the state, then $A$ is referred to as an inner attribute. In addition, a state-dependent attribute $A$ may be declared as inner attribute if, for each relevant particle, the value of $A$ is defined and constant over the whole observation period. Often a particularly convenient description of a system may be achieved by a suitable choice of the inner attributes (see Sect. 2.6.2).

\subsection{Identical and Indistinguishable Particles}

Two particles are called identical if the values of all their inner attributes agree. $\mathcal{H}$ must be so constituted that the transposition of two identical particles is defined for every vector in $\mathcal{H}$ (quantum case) or every phase space point in $\mathcal{H}$ (classical case), respectively. Two identical particles are called indistinguishable if every pure quantum state (every classical microstate) is invariant under transposition of these two particles; otherwise the two particles are called distinguishable. Two non-identical particles are always considered distinguishable.

\subsection{Identical Particles in Quantum Mechanics}

\subsubsection{Symmetrization Postulate and Indistinguishability of Identical Quantum Particles}

In quantum mechanics, the symmetrization postulate [28] claims that a state vector $|\mathbf{s}\rangle$, under the transposition $\sigma$ of two identical particles that are both system particles in $\mathrm{s}$, either stays the same (bosons) or changes by the phase factor of -1 (fermions). (The symmetrization postulate also holds if the two identical system particles are composite particles [12].) From the symmetrization postulate and (2.15) it follows that

$$
|\sigma(\mathbf{s})\rangle= \pm e^{\mathrm{i} \varphi}|\mathbf{s}\rangle \quad(\varphi \in \mathbb{R}),
$$

i.e., $|\sigma(\mathrm{s})\rangle$ and $|\mathbf{s}\rangle$ only differ by a phase factor and thus represent the same pure state

$$
\sigma(\mathrm{s})=\mathrm{s}
$$

(see Sect. 2.1.1). Hence, pure states are invariant under permutation of identical system particles.

According to (2.14) and (2.15), the transposition of a system particle with an identical particle that is not a system particle maps a pure state $s$ to a pure state in which the particle composition of the system is different from that in s. However, since those two pure states are experimentally indiscernible, they may be identified with one another. After that identification, state vectors arising from each other through permutation of identical particles (system particles or not) always represent the same pure state; identical particles are then indistinguishable. The present article adheres to this identification. (Without identifying pure states arising from each other through permutation of system particles with identical particles that are not system particles, identical particles generally would be pairwise distinguishable. Distinguishable identical quantum particles will be discussed elsewhere.) 


\subsubsection{Consequences of the Choice of the Inner Attributes}

Whether or not two particles are identical and thus obey the symmetrization postulate depends on which particle attributes are declared as inner attributes. As will be shown below by means of two examples, the choice of the inner particle attributes therefore also affects which vectors of the state space are state vectors and which particles are relevant.

As a first example, consider a system of two electrons $\mathfrak{p}_{1}$ and $\mathfrak{p}_{2}$ in the pure state $s$ : one electron is in the single-particle state $\psi$ and has $z$-spin up (i.e., the $z$-component of the spin has the value $+\frac{\hbar}{2}$ in $\psi$ ), the other is in the single-particle state $\phi$ and has $z$-spin down. Usually, $\mathfrak{p}_{1}$ and $\mathfrak{p}_{2}$ are considered identical and obey the symmetrization postulate. Since electrons are fermions, $s$ is represented by the antisymmetric state vector

$$
|\mathbf{s}\rangle=\frac{1}{\sqrt{2}}\left(\left|\mathfrak{p}_{1}: \psi\right\rangle \otimes\left|\mathfrak{p}_{2}: \phi\right\rangle-\left|\mathfrak{p}_{1}: \phi\right\rangle \otimes\left|\mathfrak{p}_{2}: \psi\right\rangle\right)
$$

If, however, the $z$-spin is declared as an inner attribute, then the two electrons are nonidentical and $s$ is represented by the state vector

$$
|\mathbf{s}\rangle=\left|\mathfrak{p}_{1}: \psi\right\rangle \otimes\left|\mathfrak{p}_{2}: \phi\right\rangle
$$

(assuming that $\mathfrak{p}_{1}$ is the electron with $z$-spin up). In the first case (in which $\mathfrak{p}_{1}$ and $\mathfrak{p}_{2}$ are identical), the vector (2.20) is not a state vector because it is not antisymmetric; in the second case (in which the $z$-spin is an inner attribute), the vector (2.19) is inadmissible because for this vector the value of the $z$-spin would be neither defined for $\mathfrak{p}_{1}$ nor for $\mathfrak{p}_{2}$ (see Sect. 2.4). Of course, physical predictions do not depend on whether or not the $z$-spin is declared as an inner attribute, and by which vectors of the state space pure states therefore must be represented. (The proof of this claim is similar to [26].)

As a second example, consider the particle attribute "localization within a certain spatial domain" with the possible values 1 (for "yes") and 0 (for "no"). The value of this attribute for a particular particle is 1 (0) if the particle is located with certainty inside (outside) the regarded spatial domain. If the "localization within the system" is declared as an inner attribute, then all particles outside the system are non-identical to all particles inside the system. In this case, relevant particles are system particles over the whole observation period with certainty (and not only with non-vanishing probability), so all particles outside the system may be ignored (provided they do not interact with the system). If, however, the "localization within the system" is not an inner attribute, then there may exist identical particles neither lying all inside nor lying all outside the system. From the symmetrization postulate (applied to an appropriate superior system) it then follows that all these identical particles are relevant. Of course, physical predictions do not depend on whether or not the "localization within the system" is declared as an inner attribute, and whether certain particles outside the system may therefore be ignored or must be accounted for [27].

\subsection{Identical Particles in Classical Mechanics}

The transposition $\sigma$ of two identical particles maps a phase space point $\widehat{m}$ to a phase space point $\sigma(\widehat{\mathrm{m}})$ which is generally different from $\widehat{\mathrm{m}}$. As long as each phase space point represents a distinct microstate (see Sect. 2.1.2), the classical microstate $\sigma(\mathrm{m})$ ), defined by (2.16), is generally different from $\mathrm{m}$. In this case, identical classical particles are pairwise distinguishable. 
Microstates arising from each other through permutation of identical particles are experimentally indiscernible [see (5.34) in Sect. 5.6.3]. Hence, these microstates may be identified with one another. After that identification, phase space points arising from each other through permutation of identical particles represent the same microstate; identical classical particles are then indistinguishable (cf. Sect. 5.6.2).

Planck [31, 32] (since about 1916) and others after him (e.g., Rushbrooke [34]) were of the opinion that experimentally indiscernible microstates must be identified with one another and that identical classical particles are therefore necessarily indistinguishable. Ehrenfest [13], Schrödinger [37] and others (e.g., Ter Haar [42]) objected to this; their contention was that classical particles are inherently distinguishable. Before Planck, Gibbs [16] already regarded identical classical particles as indistinguishable in order to avoid the GP1. (This reasoning was later supported particularly explicitly by Hestenes [19].) In the opinion of the present author, all positions just mentioned are not correct regarding their claim to absoluteness. In fact, one has the choice of assuming identical classical particles to be distinguishable or indistinguishable; in Sect. 5.6 it is shown that both possibilities are equivalent.

\section{Gibbs Paradox of the First Kind (GP1)}

\subsection{Demonstration of the GP1}

\subsubsection{Non-identical Particles}

Consider a vessel of volume $V$ containing a (quantum or classical) particle of mass $m$ without internal degrees of freedom at (not too low) temperature $T$. The canonical partition function of this system is

$$
z(T, V)=V\left(\frac{m k T}{2 \pi \hbar^{2}}\right)^{3 / 2}
$$

[35]. [For the classical case, an elementary phase space volume of $(2 \pi \hbar)^{3}$ is assumed.]

Now, let the vessel be filled with $N(N \gg 1)$ pairwise non-identical particles. For simplicity, let all $N$ particles have the same mass $m$ and no internal degrees of freedom (cf. Remark 3.2). Further, let there be no interaction between the particles; the particles then form an ideal gas with the canonical partition function

$$
Z(T, N, V)=z^{N} \stackrel{(3.1)}{=}\left[V\left(\frac{m k T}{2 \pi \hbar^{2}}\right)^{3 / 2}\right]^{N} .
$$

As a general rule, one obtains the entropy $S(T, \ldots)$ of a system in canonical equilibrium from its partition function $Z(T, \ldots)$ by

$$
S(T, \ldots)=\frac{\partial}{\partial T}(k T \ln Z)
$$

[9]. In particular, (3.2) yields

$$
S(T, N, V)=N k\left[\ln V+\frac{3}{2} \ln \frac{m k T}{2 \pi \hbar^{2}}+\frac{3}{2}\right] .
$$


$S(T, N, V)$ is not a homogeneous function of degree 1 in the variables $N$ and $V$, that is, the entropy is not extensive in the case of the ideal gas considered above. Assuming that the entropy is additive, this leads to the GP1 as follows:

A vessel of volume $V$ containing $N$ pairwise non-identical non-interacting particles of the same mass $m$ is divided into two equal subsystems $\mathbb{S}_{1}$ and $\mathbb{S}_{2}$ by inserting a partition. Thus, both subsystems have the same volume $\frac{V}{2}$ and each contains $\frac{N}{2}$ particles. (Strictly speaking, there are also other possible distributions of the $N$ particles on the two subsystems, for example, $\frac{N}{2}-1$ particles in one subsystem and $\frac{N}{2}+1$ in the other [5]. Here and in the following, this may be neglected.) The entropy of the total system consisting of $\mathbb{S}_{1}$ and $\mathbb{S}_{2}$ is additively composed of the entropies of the two subsystems and thus is $2 S\left(T, \frac{N}{2}, \frac{V}{2}\right)$. $\mathbb{S}_{1}$ and $\mathbb{S}_{2}$ can be recombined by removing the partition; the original vessel of total volume $V$ containing all $N$ particles then emerges again. Paradoxically, the removal of the partition seems to increase the entropy by

$$
S(T, N, V)-2 S\left(T, \frac{N}{2}, \frac{V}{2}\right) \stackrel{(3.4)}{=} N k \ln 2 .
$$

(The entropy increase is paradoxical for the following reason: The system is in the same macrostate after the removal of the partition as before its insertion and therefore must have the same entropy at both times. Thus, if the entropy of the system increases when removing the partition, then it must have decreased by the same amount before, when the partition was inserted. However, a decrease in system entropy when inserting the partition contradicts the second law of thermodynamics because the insertion of the partition is an adiabatic process.)

Remark 3.1 Actually, it is not necessary to remove the partition in order for a paradox to arise, since, as can be shown similar to above, the insertion of the partition seems to decrease the entropy by $N k \ln 2$ and thus already yields a contradiction to the second law of thermodynamics. In the literature, however, the GP1 is always associated with the entropy increase when combining two systems and not with the entropy decrease when dividing a system; for that reason, the GP1 is also here presented in this conventional form.

Remark 3.2 One might object that particles having the same mass and no internal degrees of freedom must be identical. This objection can be dealt with by assuming that the $N$ particles have pairwise different masses lying all so close to $m$ that the entropy of the gas deviates only negligibly (i.e., considerably less than $N k \ln 2$ ) from (3.4).

Remark 3.3 In Sect. 4, it is shown that the formulas (3.1), (3.2) and, as a consequence, (3.4) only hold if there is no uncertainty about which particle is (about which $N$ particles are) located in the vessel.

Remark 3.4 A more extreme variant of the GP1 in which an irreversible process seems to lead to an entropy decrease is described by Swendsen in [41].

\subsubsection{Distinguishable Identical Particles}

Since this article adheres to the indistinguishability of identical quantum particles (see Sect. 2.6.1), distinguishable identical particles necessarily are classical particles. If there are $N$ distinguishable identical classical particles in the vessel, then one obtains the same entropy (3.4) as for pairwise non-identical particles of equal mass (the derivation is the same as in Sect. 3.1.1). Thus, the GP1 arises for distinguishable identical classical particles as well. 


\subsubsection{Indistinguishable Particles}

If the $N$ particles in the vessel are indistinguishable, then, compared to the non-identical particles regarded in Sect. 3.1.1, each $N$ ! microstates arising from each other through permutation of the particles in the vessel coincide to one single microstate. Thus, for indistinguishable particles, the canonical partition function $\widetilde{Z}$ is $N$ ! times smaller than in (3.2):

$$
\widetilde{Z}(T, N, V)=\frac{1}{N !} Z \stackrel{(3.2)}{=} \frac{1}{N !}\left[V\left(\frac{m k T}{2 \pi \hbar^{2}}\right)^{3 / 2}\right]^{N}
$$

[In the quantum case, the particle density is assumed to be so low and/or the temperature so high that microstates with multiply occupied single-particle states only negligibly contribute to the logarithm of the partition function [1]; otherwise the particles must be described by Bose-Einstein or Fermi-Dirac statistics. In the classical case, there is no such restriction of the scope of application of (3.6) regarding pressure and temperature because the set of microstates with multiple occupancies, that is, the set of microstates for which at least two particles have the same generalized coordinates and momenta, has measure zero.] The entropy

$$
\begin{gathered}
\widetilde{S}(T, N, V) \stackrel{(3.3),(3.6)}{=} N k\left[\ln V+\frac{3}{2} \ln \frac{m k T}{2 \pi \hbar^{2}}+\frac{3}{2}\right]-k \ln N ! \\
\stackrel{\text { Stirling }}{\approx} N k\left[\ln \frac{V}{N}+\frac{3}{2} \ln \frac{m k T}{2 \pi \hbar^{2}}+\frac{5}{2}\right]
\end{gathered}
$$

resulting from $\widetilde{Z}$ (Sackur-Tetrode equation) is extensive; thus, for indistinguishable particles the GP1 does not arise.

Remark 3.5 In the derivation above, the partition function (3.6) and consequently the Sackur-Tetrode equation (3.7) are ultimately based on the partition function (3.2), which is generally not correct (cf. Remark 3.3). However, the Sackur-Tetrode equation is still correct for indistinguishable particles, as one may verify in the classical case by plugging (6.2) into (5.60).

\subsection{Physical Origin of the GP1}

In order to attain a resolution of the GP1, it is useful to first illuminate the origin of the paradox. For that purpose, consider again an ideal gas of $N(N \gg 1)$ pairwise non-identical (quantum or classical) particles of the same mass $m$ without internal degrees of freedom (cf. Remark 3.2). Let there be no uncertainty about of which $N$ particles the ideal gas consists (cf. Remark 3.3) and let the gas be confined to a vessel of volume $V$ at temperature $T$. This vessel is then divided into two equal subsystems $\mathbb{S}_{1}$ and $\mathbb{S}_{2}\left(N_{1}=N_{2}=\frac{N}{2}, V_{1}=V_{2}=\frac{V}{2}\right)$ by inserting a partition. According to (3.4), the entropy $S_{\mathrm{p}}$ of the gas previous to the partitioning is

$$
S_{\mathrm{p}}=S(T, N, V)=N k\left[\ln V+\frac{3}{2} \ln \frac{m k T}{2 \pi \hbar^{2}}+\frac{3}{2}\right] .
$$

Let $S_{\mathrm{a}}$ be the entropy of the total system consisting of $\mathbb{S}_{1}$ and $\mathbb{S}_{2}$ after the partitioning; let $S_{1}$ and $S_{2}$ be the entropies of the subsystems $\mathbb{S}_{1}$ and $\mathbb{S}_{2}$. The GP1 arises if one calculates $S_{1}$ 
and $S_{2}$ using (3.4) and then sums these two entropies in order to obtain $S_{\mathrm{a}}$ :

$$
\begin{aligned}
S_{\mathrm{a}} & \stackrel{?}{=} S_{1}+S_{2} \stackrel{?}{=} S\left(T, N_{1}, V_{1}\right)+S\left(T, N_{2}, V_{2}\right)=2 S\left(T, \frac{N}{2}, \frac{V}{2}\right) \\
& \stackrel{(3.4)}{=} N k\left[\ln V+\frac{3}{2} \ln \frac{m k T}{2 \pi \hbar^{2}}+\frac{3}{2}\right]-N k \ln 2 .
\end{aligned}
$$

Comparison with $S_{\mathrm{p}}$ yields an entropy decrease (!) of

$$
S_{\mathrm{p}}-S_{\mathrm{a}} \stackrel{?}{=} N k \ln 2
$$

from the partitioning.

However, the calculation (3.9) is only correct if one knows which $\frac{N}{2}$ of the $N$ particles have landed in $\mathbb{S}_{1}$ and which in $\mathbb{S}_{2}$. In fact, there is no information about this (as long as no relevant measurement is performed). Hence, after the partitioning, a complete counting of the possible microstates of the total system consisting of $\mathbb{S}_{1}$ and $\mathbb{S}_{2}$ must take into account all $\left(\begin{array}{c}N \\ N / 2\end{array}\right)$ possibilities of how the $N$ particles can be distributed over the two subsystems. This implies a canonical partition function that is larger by a factor of $\left(\begin{array}{c}N \\ N / 2\end{array}\right)$ [47], and thus, according to (3.3), an entropy $S_{\mathrm{a}}$ that is larger by

$$
\begin{aligned}
k \ln \left(\begin{array}{c}
N \\
N / 2
\end{array}\right) & =k\left[\ln N !-2 \ln \frac{N}{2} !\right] \stackrel{\text { Stirling }}{\approx} k\left[N \ln N-N-2\left(\frac{N}{2} \ln \frac{N}{2}-\frac{N}{2}\right)\right] \\
& =N k \ln 2
\end{aligned}
$$

than the entropy $S_{\mathrm{a}}$ calculated in (3.9), that is,

$$
S_{\mathrm{a}}=S_{\mathrm{p}}
$$

Hence, as expected, the entropy is not changed by the partitioning.

One recognizes that the origin of the GP1 lies in the incomplete counting of the possible microstates: in the calculation (3.9), the uncertainty about the identities of the particles located in $\mathbb{S}_{1}\left(\mathbb{S}_{2}\right.$, respectively) was disregarded. [The uncertainty about the number of the particles in $\mathbb{S}_{1}\left(\mathbb{S}_{2}\right.$, respectively) was disregarded as well by the assumption $N_{1}=N_{2}=\frac{N}{2}$. However, this uncertainty (as opposed to the previous one) only negligibly contributes to $S_{\mathrm{a}}$; its non-consideration is compensated by the Stirling approximation in (3.11) [5].] Thus, the GP1 and its origin show that, for the statistical treatment of a system of non-identical particles (Sect. 4), one has to take into account that the possible microstates may also differ with respect to the particle composition of the system. [The same holds for systems of distinguishable identical classical particles (Sect. 5).]

Remark 3.6 In the equation chain (3.9), neither the first nor the second equals sign tagged by a question mark is correct. For the entropy $S_{1}$ of $\mathbb{S}_{1}$ it holds that

$$
S_{1} \stackrel{(3.11)}{=} S\left(T, N_{1}, V_{1}\right)+N k \ln 2,
$$

because there are $\left(\begin{array}{c}N \\ N / 2\end{array}\right)$ possibilities of which particles can be located in $\mathbb{S}_{1}$ (see Sect. 4.3.1). Correspondingly, it holds that

$$
S_{2}=S\left(T, N_{2}, V_{2}\right)+N k \ln 2 .
$$


Further, the entropy $S_{\mathrm{a}}$ of the total system is not the sum of the entropies of the two subsystems because the two subsystems are not independent of each other (see Sect. 4.3.2.4). Namely, $\mathbb{S}_{2}$ contains exactly those $\frac{N}{2}$ particles that are not located in $\mathbb{S}_{1}$.

\section{Statistics of Non-identical Particles}

Let a set $\mathcal{P}$ of $\mathfrak{N}$ pairwise non-identical (quantum or classical) particles be given. The elements of $\mathcal{P}$ are referred to as "particles of the kind $\mathcal{P}$ " or " $\mathcal{P}$-particles" for short. Further, let a system $\mathbb{S}$ of $N(N \leq \mathfrak{N}) \mathcal{P}$-particles be given that is closed with respect to particle exchange. From the closedness of $\mathbb{S}$ does not follow that there is certainty of which $N$ of the $\mathfrak{N} \mathcal{P}$-particles $\mathbb{S}$ is composed. Only a change in the particle composition of $\mathbb{S}$ is prevented by the closedness. More precisely, a change of the probabilities of the possible compositions of $\mathbb{S}$ is prevented (see Sect. 4.2.1). [However, a change of these probabilities is still possible despite the closedness of $\mathbb{S}$ if a measurement is performed on $\mathbb{S}$ or on a system correlated with $\mathbb{S}$. If, for example, a certain $\mathcal{P}$-particle $\mathfrak{p}$ first is a system particle of $\mathbb{S}$ with a non-vanishing probability, and at a later point in time a measurement on another system shows that $\mathfrak{p}$ is located in that other system with certainty, then the probability of $\mathfrak{p}$ being a system particle of $\mathbb{S}$ collapses to zero at the time of that measurement. This is the EPR-effect [14], which, in the form just described, also arises in classical statistical mechanics.]

In the following, (particle) compositions of systems are represented by sets of particles: if, for example, $\mathbb{S}$ is composed of the particles $\mathfrak{p}_{1}, \mathfrak{p}_{2}, \ldots, \mathfrak{p}_{N} \in \mathcal{P}$, then $\left\{\mathfrak{p}_{1}, \mathfrak{p}_{2}, \ldots, \mathfrak{p}_{N}\right\} \subset \mathcal{P}$ is the composition of $\mathbb{S}$. The elements of a composition $\zeta$ are referred to as " $\zeta$-particles". For a pure quantum state or a classical microstate $\mathrm{m}$, the phrasings " $\mathrm{S}$ has in $\mathrm{m}$ the composition $\zeta$ " or " $m$ belongs to the composition $\zeta$ " mean that the $\zeta$-particles (and no other particles) are system particles in $\mathrm{m}$.

\subsection{Entropy of a Macrostate}

\subsubsection{Quantum System}

Let the (quantum) system $\mathbb{S}$ be in the macrostate $M$. Let $\left.\zeta_{1}, \zeta_{2}, \ldots, \zeta_{(}^{\mathfrak{N}}\right)$ be the $\left(\begin{array}{l}\mathfrak{N} \\ N\end{array}\right)$ compositions of $\mathbb{S}$ consisting of $N \mathcal{P}$-particles. Let $\mathcal{M}$ be the set of microstates of $\mathrm{M}$ and $\mu_{i} \subset \mathcal{M}$ the set of those microstates of $\mathrm{M}$ that belong to the composition $\zeta_{i}$. It holds that

$$
\mu_{i} \cap \mu_{j}=\emptyset \quad(i \neq j) .
$$

According to Sect. 2.1.1, each microstate of $\mathrm{M}$ belongs to a certain composition. Since $\mathbb{S}$, by hypothesis, consists of $N \mathcal{P}$-particles, only the compositions $\zeta_{1}, \zeta_{2}, \ldots, \zeta_{\left(\begin{array}{l}\mathfrak{N} \\ N\end{array}\right)}$ are admissible for $\mathbb{S}$, that is, microstates belonging to another composition possess the probability zero in M. Thus, without loss of generality,

$$
\mathcal{M}=\bigcup_{i=1}^{\left(\begin{array}{c}
\mathfrak{N} \\
N
\end{array}\right)} \mu_{i}
$$

can be assumed from the outset.

Let $P_{\mathrm{M}}(\mathrm{m})$ be the probability of the microstate $\mathrm{m} \in \mathcal{M}$ in the macrostate $\mathrm{M}$. The probability $P_{\mathrm{M}}\left(\zeta_{i}\right)$ that $\mathbb{S}$ has the composition $\zeta_{i}$ in $\mathrm{M}$ is

$$
P_{\mathrm{M}}\left(\zeta_{i}\right)=\sum_{\mathrm{m} \in \mu_{i}} P_{\mathrm{M}}(\mathrm{m})
$$


It holds that

$$
\sum_{i=1}^{\left(\begin{array}{c}
\mathfrak{N} \\
N
\end{array}\right)} P_{\mathrm{M}}\left(\zeta_{i}\right) \stackrel{(4.3)}{=} \sum_{i=1}^{\left(\begin{array}{c}
\mathfrak{N} \\
N
\end{array}\right)} \sum_{\mathrm{m} \in \mu_{i}} P_{\mathrm{M}}(\mathrm{m}) \stackrel{(4.1),(4.2)}{=} \sum_{\mathrm{m} \in \mathcal{M}} P_{\mathrm{M}}(\mathrm{m}) \stackrel{(2.5)}{=} 1 .
$$

Further, let $P_{\mathrm{M}}\left(\mathrm{m} \mid \zeta_{i}\right)$ be the conditional probability of the microstate $\mathrm{m}$ in the macrostate $\mathrm{M}$, given that $\mathbb{S}$ has the composition $\zeta_{i}$, i.e.,

$$
P_{\mathrm{M}}\left(\mathrm{m} \mid \zeta_{i}\right)=\left\{\begin{array}{ll}
\frac{P_{\mathrm{M}}(\mathrm{m})}{P_{\mathrm{M}}\left(\zeta_{i}\right)} & \text { for } \mathrm{m} \in \mu_{i} \\
0 & \text { for } \mathrm{m} \notin \mu_{i}
\end{array} \quad\left(P_{\mathrm{M}}\left(\zeta_{i}\right) \neq 0\right) .\right.
$$

For $P_{\mathrm{M}}\left(\zeta_{i}\right) \neq 0$, it holds that

$$
\sum_{\mathrm{m} \in \mu_{i}} P_{\mathrm{M}}\left(\mathrm{m} \mid \zeta_{i}\right) \stackrel{(4.5)}{=} \sum_{\mathrm{m} \in \mu_{i}} \frac{P_{\mathrm{M}}(\mathrm{m})}{P_{\mathrm{M}}\left(\zeta_{i}\right)} \stackrel{(4.3)}{=} 1
$$

For $P_{\mathrm{M}}\left(\zeta_{i}\right) \neq 0$, let the conditional entropy $S\left(\mathrm{M} \mid \zeta_{i}\right)$ of $\mathrm{M}$ with respect to $\zeta_{i}$ be defined as

$$
S\left(\mathrm{M} \mid \zeta_{i}\right):=-k \sum_{\mathrm{m} \in \mu_{i}} P_{\mathrm{M}}\left(\mathrm{m} \mid \zeta_{i}\right) \ln P_{\mathrm{M}}\left(\mathrm{m} \mid \zeta_{i}\right)
$$

With this, one obtains for the entropy $S$ of M,

$$
S(\mathrm{M})=\sum_{i=1}^{\left(\begin{array}{c}
\mathfrak{N} \\
N
\end{array}\right)} P_{\mathrm{M}}\left(\zeta_{i}\right) S\left(\mathrm{M} \mid \zeta_{i}\right)-k \sum_{i=1}^{\left(\begin{array}{c}
\mathfrak{N} \\
N
\end{array}\right)} P_{\mathrm{M}}\left(\zeta_{i}\right) \ln P_{\mathrm{M}}\left(\zeta_{i}\right)
$$

Proof It holds that

$$
\begin{aligned}
& S(\mathrm{M}) \stackrel{(2.7)}{=}-k \sum_{\mathrm{m} \in \mathcal{M}} P_{\mathrm{M}}(\mathrm{m}) \ln P_{\mathrm{M}}(\mathrm{m}) \\
& \stackrel{(4.1),(4.2)}{=}-k \sum_{i=1}^{(4.5)} \sum_{\mathrm{m} \in \mu_{i}} P_{\mathrm{M}}(\mathrm{m}) \ln \left[P_{\mathrm{M}}\left(\mathrm{m} \mid \zeta_{i}\right) P_{\mathrm{M}}\left(\zeta_{i}\right)\right] \\
& \stackrel{(4.5)}{=}-k \sum_{i=1}^{\left(\begin{array}{c}
\mathfrak{N} \\
N
\end{array}\right)} \sum_{\mathrm{m} \in \mu_{i}} P_{\mathrm{M}}\left(\zeta_{i}\right) P_{\mathrm{M}}\left(\mathrm{m} \mid \zeta_{i}\right) \ln P_{\mathrm{M}}\left(\mathrm{m} \mid \zeta_{i}\right)-k \sum_{i=1}^{\left(\begin{array}{c}
\mathfrak{N} \\
N
\end{array}\right)} \sum_{\mathrm{m} \in \mu_{i}} P_{\mathrm{M}}(\mathrm{m}) \ln P_{\mathrm{M}}\left(\zeta_{i}\right) \\
& \stackrel{(4.7),(4.3)}{=} \sum_{i=1}^{\left(\begin{array}{c}
\mathfrak{N} \\
N
\end{array}\right)} P_{\mathrm{M}}\left(\zeta_{i}\right) S\left(\mathrm{M} \mid \zeta_{i}\right)-k \sum_{i=1}^{\left(\begin{array}{c}
\mathfrak{N} \\
N
\end{array}\right)} P_{\mathrm{M}}\left(\zeta_{i}\right) \ln P_{\mathrm{M}}\left(\zeta_{i}\right)
\end{aligned}
$$

$\left[P_{\mathrm{M}}\left(\zeta_{i}\right) P_{\mathrm{M}}\left(\mathrm{m} \mid \zeta_{i}\right)\right.$ and $P_{\mathrm{M}}\left(\zeta_{i}\right) S\left(\mathrm{M} \mid \zeta_{i}\right)$ are set to zero for $\left.P_{\mathrm{M}}\left(\zeta_{i}\right)=0.\right]$

Thus, the entropy of $\mathrm{M}$ is composed of two contributions: the weighted arithmetic mean of the conditional entropies and a contribution originating from the uncertainty about the composition of $\mathbb{S}$. 
Remark 4.1 $P_{\mathrm{M}}\left(\zeta_{i}\right)$ is well-defined, that is, independent of the choice of the microstates, since, according to Sect. 2.1.1, $P_{\mathrm{M}}\left(\zeta_{i}\right)$ can be written as

$$
P_{\mathrm{M}}\left(\zeta_{i}\right) \stackrel{(4.3)}{=} \operatorname{Tr}\left\{\rho \Pi_{i}\right\}
$$

where $\rho$ is the density operator representing $\mathrm{M}, \Pi_{i}$ is the orthogonal projection onto the many-particle state space of the $\zeta_{i}$-particles and $\operatorname{Tr}\{\ldots\}$ stands for "trace of ...". The well-definedness of $S\left(\mathrm{M} \mid \zeta_{i}\right)$ can be proven similarly to the well-definedness of $P_{\mathrm{M}}\left(\zeta_{i}\right)$ and $S(\mathrm{M})[8]$.

\subsubsection{Classical System}

Let the (classical) system $\mathbb{S}$ be in the macrostate M. Let $\left.\zeta_{1}, \zeta_{2}, \ldots, \zeta_{(\mathfrak{N}}^{\mathfrak{N}}\right)$ be the compositions of $\mathbb{S}$ consisting of $N \mathcal{P}$-particles and $\mathcal{U}_{i}$ the phase space spanned by the generalized coordinates and momenta of the $\zeta_{i}$-particles. Since $\mathbb{S}$, by hypothesis, consists of $N \mathcal{P}$-particles, only the compositions $\zeta_{1}, \zeta_{2}, \ldots, \zeta_{\left(\begin{array}{l}\mathfrak{N} \\ N\end{array}\right)}$ are admissible for $\mathbb{S}$, that is, phase space points not lying in one of the phase spaces $\mathcal{U}_{1}, \mathcal{U}_{2}, \ldots, \mathcal{U}_{\left(\begin{array}{l}\mathfrak{N} \\ N\end{array}\right)}$ possess the probability density zero in $\mathrm{M}$. Thus,

$$
\mathcal{H}:=\bigcup_{i=1}^{\left(\begin{array}{c}
\mathfrak{N} \\
N
\end{array}\right)} \mathcal{U}_{i}
$$

can be taken as the set of all phase space points of $\mathbb{S}$ from the outset (cf. Sect. 2.1.2).

Let $\rho_{\mathrm{M}}(\widehat{\mathrm{m}})$ be the probability density of the phase space point $\widehat{\mathrm{m}} \in \mathcal{H}$ in the macrostate $\mathrm{M}$. The probability $P_{\mathrm{M}}\left(\zeta_{i}\right)$ that $\mathbb{S}$ has the composition $\zeta_{i}$ in $\mathrm{M}$ is

$$
P_{\mathrm{M}}\left(\zeta_{i}\right)=\int \mathrm{d} \mathcal{U}_{i} \rho_{\mathrm{M}}(\widehat{\mathrm{m}})
$$

It holds that

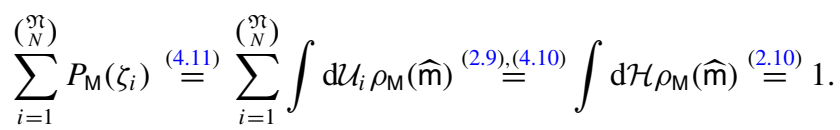

Further, let $\rho_{\mathrm{M}}\left(\widehat{\mathrm{m}} \mid \zeta_{i}\right)$ be the conditional probability density of the phase space point $\widehat{\mathrm{m}}$ in the macrostate $\mathrm{M}$, given that $\mathbb{S}$ has the composition $\zeta_{i}$, i.e.,

$$
\rho_{\mathrm{M}}\left(\widehat{\mathrm{m}} \mid \zeta_{i}\right)=\left\{\begin{array}{ll}
\frac{\rho_{\mathrm{M}}(\widehat{\mathrm{m}})}{P_{\mathrm{M}}\left(\zeta_{i}\right)} & \text { for } \widehat{\mathrm{m}} \in \mathcal{U}_{i} \\
0 & \text { for } \widehat{\mathrm{m}} \notin \mathcal{U}_{i}
\end{array} \quad\left(P_{\mathrm{M}}\left(\zeta_{i}\right) \neq 0\right) .\right.
$$

For $P_{\mathrm{M}}\left(\zeta_{i}\right) \neq 0$, it holds that

$$
\int \mathrm{d} \mathcal{U}_{i} \rho_{\mathrm{M}}\left(\widehat{\mathrm{m}} \mid \zeta_{i}\right) \stackrel{(4.13)}{=} \int \mathrm{d} \mathcal{U}_{i} \frac{\rho_{\mathrm{M}}(\widehat{\mathrm{m}})}{P_{\mathrm{M}}\left(\zeta_{i}\right)} \stackrel{(4.11)}{=} 1
$$

For $P_{\mathrm{M}}\left(\zeta_{i}\right) \neq 0$, let the conditional entropy $S\left(\mathrm{M} \mid \zeta_{i}\right)$ of $\mathrm{M}$ with respect to $\zeta_{i}$ be defined as

$$
S\left(\mathrm{M} \mid \zeta_{i}\right):=-k \int \mathrm{d} \mathcal{U}_{i} \rho_{\mathrm{M}}\left(\widehat{\mathrm{m}} \mid \zeta_{i}\right) \ln \left[\rho_{\mathrm{M}}\left(\widehat{\mathrm{m}} \mid \zeta_{i}\right)(2 \pi \hbar)^{\frac{\mathrm{dim} \mathcal{U}_{i}}{2}}\right]
$$


With this, one obtains for the entropy $S$ of $\mathrm{M}$, as in the quantum case,

$$
S(\mathrm{M})=\sum_{i=1}^{\left(\begin{array}{c}
\mathfrak{N} \\
N
\end{array}\right)} P_{\mathrm{M}}\left(\zeta_{i}\right) S\left(\mathrm{M} \mid \zeta_{i}\right)-k \sum_{i=1}^{\left(\begin{array}{c}
\mathfrak{N} \\
N
\end{array}\right)} P_{\mathrm{M}}\left(\zeta_{i}\right) \ln P_{\mathrm{M}}\left(\zeta_{i}\right)
$$

The proof of (4.16) is similar to the proof of (4.8) in Sect. 4.1.1 and is therefore not repeated here.

In the remainder of this section, only quantum systems will be considered. However, all results also hold for classical systems; the proofs are similar to their quantum analogs.

\subsection{Equilibrium State}

Now, let the system $\mathbb{S}$ be isolated, i.e., closed also with respect to energy exchange. Let $\mathbb{S}$ in the equilibrium state $Q$ possess the fixed energy $E$, that is, all microstates in which the energy of the system deviates (more than an inaccuracy $\delta E$ ) from $E$ possess the probability zero in $\mathrm{Q}$. (An equilibrium state is a macrostate that is steady with respect to the second law of thermodynamics.) Again, let $\zeta_{1}, \zeta_{2}, \ldots, \zeta_{\left(\begin{array}{l}\mathfrak{N} \\ N\end{array}\right)}$ be the compositions of $\mathbb{S}$ consisting of $N$ $\mathcal{P}$-particles, $\mathcal{M}$ the set of microstates of $\mathrm{Q}$ and $\mu_{i} \subset \mathcal{M}$ the set of those microstates of $\mathrm{Q}$ that belong to the composition $\zeta_{i}$. Let $\mu_{i}^{*} \subset \mu_{i}$ be the set of microstates of $\mathrm{Q}$ that belong to $\zeta_{i}$ and are a priori possible. (A microstate of $\mathrm{Q}$ is referred to as a priori possible if its energy lies between $E-\delta E$ and $E+\delta E$.)

\subsubsection{Composition Probabilities}

The fundamental postulate of statistical mechanics states that, for an isolated system with fixed energy and particle number in equilibrium (microcanonical equilibrium), all a priori possible microstates are equiprobable. However, the fundamental postulate cannot directly be applied to $\mathbb{S}$ because it implicitly assumes an ergodic system [10] and $\mathbb{S}$ is generally not ergodic. Namely, a priori possible microstates belonging to different compositions cannot pass into one another due to the closedness of $\mathbb{S}$ with respect to particle exchange.

A priori possible microstates belonging to the same composition $\zeta_{i}$, on the other hand, can pass into one another (unless $\mathbb{S}$ is subject to internal constraints; cf. Remark 4.7). Hence, according to the fundamental postulate (in restricted form), these microstates have the same probability in equilibrium. Thus, because of (4.3), it holds that

$$
P_{\mathrm{Q}}(\mathrm{m})=\left\{\begin{array}{ll}
P_{\mathrm{Q}}\left(\zeta_{i}\right) /\left|\mu_{i}^{*}\right| & \text { for } \mathrm{m} \in \mu_{i}^{*} \\
0 & \text { for } \mathrm{m} \in \mu_{i} \backslash \mu_{i}^{*} ;
\end{array} \quad i=1,2, \ldots,\left(\begin{array}{l}
\mathfrak{N} \\
N
\end{array}\right) .\right.
$$

The $\left|\mu_{i}^{*}\right|\left(i=1,2, \ldots,\left(\begin{array}{l}\mathfrak{N} \\ N\end{array}\right)\right)$ in (4.17) are determined by the compositions $\zeta_{1}, \zeta_{2}$, $\ldots, \zeta_{\left(\begin{array}{l}\mathfrak{N} \\ N\end{array}\right)}$, by the energy $E$ and, as the case may be, by the values of the alterable system parameters (for example, the volume or an external electric field may be alterable); however, the composition probabilities $P_{\mathrm{Q}}\left(\zeta_{1}\right), P_{\mathrm{Q}}\left(\zeta_{2}\right), \ldots, P_{\mathrm{Q}}\left(\zeta_{\left(\begin{array}{c}\mathfrak{N} \\ N\end{array}\right)}\right)$ are still undetermined. These probabilities depend on the exact circumstances during the preparation of the system. [Since the $\mathcal{P}$-particles are pairwise non-identical, certain $\mathcal{P}$-particles may preferentially have entered the system during the system preparation due to their specific properties. Hence, compositions containing these preferred $\mathcal{P}$-particles have an increased probability at the time of the isolation (see Sect. 6.2.3 for an example). Then, from the onset of the isolation, the composition probabilities are "frozen" because microstates belonging to different compositions are no longer able to pass into one another.] Thus, it holds that: 
Theorem 4.2 In addition to the energy $E$ and, if any, the values of the alterable system parameters, the complete characterization of a microcanonical equilibrium state $\mathrm{Q}$ of $\mathbb{S}$ requires the composition probabilities $P_{\mathrm{Q}}\left(\zeta_{1}\right), P_{\mathrm{Q}}\left(\zeta_{2}\right), \ldots, P_{\mathrm{Q}}\left(\zeta_{\left(\begin{array}{l}\mathfrak{N} \\ N\end{array}\right)}\right)$.

Remark 4.3 Just as an isolated system does not need to have a fixed particle composition, it also does not need to have a fixed energy or particle number. (Otherwise, for example, the adiabatic process of dividing an isolated system into two isolated subsystems by inserting an insulating partition would lead to an entropy decrease $[5,15]$ contradicting the second law of thermodynamics.) For instance, an isolated system may very well be in a macrostate whose microstates possess canonically distributed probabilities. (Without justification, the opposite assertion is made, e.g., in [45].) For $\mathbb{S}$, the fixed particle number $N$ and in this section (Sect. 4.2) the fixed energy $E$ therefore were explicitly assumed.

\subsubsection{Conditional Entropies}

For $P_{\mathrm{Q}}\left(\zeta_{i}\right) \neq 0$, the conditional entropy of $\mathrm{Q}$ with respect to $\zeta_{i}$ is

$$
\begin{gathered}
S\left(\mathrm{Q} \mid \zeta_{i}\right) \stackrel{(4.7),(4.5)}{=}-k \sum_{\mathrm{m} \in \mu_{i}} \frac{P_{\mathrm{Q}}(\mathrm{m})}{P_{\mathrm{Q}}\left(\zeta_{i}\right)} \ln \frac{P_{\mathrm{Q}}(\mathrm{m})}{P_{\mathrm{Q}}\left(\zeta_{i}\right)} \stackrel{(4.17)}{=}-k \sum_{\mathrm{m} \in \mu_{i}^{*}} \frac{1}{\left|\mu_{i}^{*}\right|} \ln \frac{1}{\left|\mu_{i}^{*}\right|} \\
=k \ln \left|\mu_{i}^{*}\right| .
\end{gathered}
$$

Let $\mathbb{S}$ in the microcanonical equilibrium state $\mathbf{Q}^{(i)}$ have the composition $\zeta_{i}$ with certainty, i.e.,

$$
P_{\mathbf{Q}^{(i)}}\left(\zeta_{j}\right)=\delta_{i j}
$$

Let the energy and the values of the alterable system parameters for $\mathrm{Q}^{(i)}$ be the same as for $\mathrm{Q}$. Thus, the same microstates can be chosen for $\mathrm{Q}^{(i)}$ as for $\mathrm{Q}$; it then holds that

$$
P_{\mathrm{Q}^{(i)}}(\mathrm{m}) \stackrel{(4.17),(4.19)}{=} \begin{cases}1 /\left|\mu_{i}^{*}\right| & \text { for } \mathrm{m} \in \mu_{i}^{*} \\ 0 & \text { otherwise. }\end{cases}
$$

Hence, the entropy of $Q^{(i)}$ is

$$
\begin{aligned}
S\left(\mathrm{Q}^{(i)}\right) & \stackrel{(2.7)}{=}-k \sum_{\mathrm{m} \in \mathcal{M}} P_{\mathrm{Q}^{(i)}}(\mathrm{m}) \ln P_{\mathrm{Q}^{(i)}}(\mathrm{m}) \stackrel{(4.1),(4.2)}{=}-k \sum_{j=1}^{(\stackrel{\mathfrak{N}}{N})} \sum_{\mathrm{m} \in \mu_{j}} P_{\mathrm{Q}^{(i)}}(\mathrm{m}) \ln P_{\mathrm{Q}^{(i)}}(\mathrm{m}) \\
& \stackrel{(4.20)}{=} k \ln \left|\mu_{i}^{*}\right| .
\end{aligned}
$$

Comparison of (4.18) with (4.21) shows:

Theorem 4.4 The conditional entropy of a microcanonical equilibrium state $Q$ with respect to a composition $\zeta_{i}$ [for which $P_{\mathrm{Q}}\left(\zeta_{i}\right) \neq 0$ ] equals the entropy of that microcanonical equilibrium state which agrees with $\mathrm{Q}$ regarding the energy and the values of the alterable system parameters, but in which the composition $\zeta_{i}$ is certain.

Theorem 4.4, derived for an isolated system with fixed energy, also holds in the canonical case (if "energy" is replaced by "temperature"). The proof of this is similar to the microcanonical case and is therefore not repeated here. 


\subsection{Harmonic Macrostates}

Again, let $\mathbb{S}$ be a system of $N \mathcal{P}$-particles that is closed with respect to particle exchange (but not necessarily isolated). A macrostate $M$ of $\mathbb{S}$ will be called $\mathcal{P}$-harmonic if in $\mathrm{M}$ all particle compositions consisting of $N \mathcal{P}$-particles are equiprobable, that is, if

$$
P_{\mathrm{M}}\left(\zeta_{1}\right)=P_{\mathrm{M}}\left(\zeta_{2}\right)=\cdots=P_{\mathrm{M}}\left(\zeta_{\left(\begin{array}{c}
\mathfrak{N} \\
N
\end{array}\right)}\right) \stackrel{(4.4)}{=} \frac{1}{\left(\begin{array}{l}
\mathfrak{N} \\
N
\end{array}\right)} .
$$

(Macrostates of systems of $\mathcal{P}$-particles whose particle number is not fixed are called $\mathcal{P}$ harmonic if compositions consisting of the same number of $\mathcal{P}$-particles are equiprobable.)

\subsubsection{Entropy of a $\mathcal{P}$-harmonic Macrostate}

According to (4.8) and (4.22), the entropy of a $\mathcal{P}$-harmonic macrostate $\mathrm{M}$ of $\mathbb{S}$ is

$$
S(\mathrm{M})=\bar{S}(\mathrm{M})+k \ln \left(\begin{array}{c}
\mathfrak{N} \\
N
\end{array}\right)
$$

where

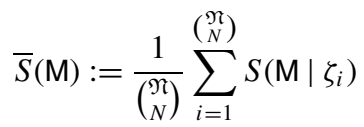

is the arithmetic mean of all conditional entropies of $\mathrm{M}$.

\subsubsection{Composite System}

Now, let two systems $\mathbb{S}_{1}$ and $\mathbb{S}_{2}$ of $N_{1}$ and $N_{2}\left(N_{1}+N_{2} \leq \mathfrak{N}\right) \mathcal{P}$-particles be given that are closed with respect to particle exchange. Let $\mathbb{S}_{1}$ and $\mathbb{S}_{2}$ be in the $\mathcal{P}$-harmonic macrostates $M_{1}$ and $M_{2}$. Except for the following condition, let there be no further correlation between $\mathbb{S}_{1}$ and $\mathbb{S}_{2}$.

Condition 4.5 A particle cannot be located in $\mathbb{S}_{1}$ with certainty while at the same time being located in $\mathbb{S}_{2}$ with certainty.

Let $\zeta_{1}, \zeta_{2}, \ldots, \zeta_{\left(N_{1}^{\mathfrak{N}}\right)}$ be the compositions of $\mathbb{S}_{1}$ consisting of $N_{1} \mathcal{P}$-particles. Let $\mathcal{M}_{1}$ be the set of microstates of $\mathrm{M}_{1}$ and $\mu_{i} \subset \mathcal{M}_{1}$ the set of those microstates of $\mathrm{M}_{1}$ that belong to $\zeta_{i}$. Let $\zeta(\mathrm{m})$ be the composition to which $\mathrm{m} \in \mathcal{M}_{1}$ belongs. Correspondingly, let $\eta_{1}, \eta_{2}, \ldots, \eta_{\left(\begin{array}{c}\mathfrak{N} \\ N_{2}\end{array}\right)}$ be the compositions of $\mathbb{S}_{2}$ consisting of $N_{2} \mathcal{P}$-particles, $\mathcal{M}_{2}$ the set of microstates of $\mathrm{M}_{2}$ and $v_{j} \subset \mathcal{M}_{2}$ the set of those microstates of $\mathrm{M}_{2}$ that belong to $\eta_{j}$. Let $\eta(\mathrm{n})$ be the composition to which $n \in \mathcal{M}_{2}$ belongs.

Let $\mathbb{S}_{1+2}$ be the total system consisting of $\mathbb{S}_{1}$ and $\mathbb{S}_{2}$, and $\theta_{1}, \theta_{2}, \ldots, \theta_{\left(N_{1}+N_{2}\right)}$ the compositions of $\mathbb{S}_{1+2}$ consisting of $N_{1}+N_{2} \mathcal{P}$-particles.

4.3.2.1 State Space of the Total System Let $\mathcal{U}_{1, i}$ be the many-particle state space of the $\zeta_{i}$ particles that belongs to $\mathbb{S}_{1}, \mathcal{U}_{2, j}$ the many-particle state space of the $\eta_{j}$-particles that belongs to $\mathbb{S}_{2}$ and $\mathcal{U}_{1+2, l}$ the many-particle state space of the $\theta_{l}$-particles that belongs to $\mathbb{S}_{1+2}$. Since, 
by hypothesis, $\mathbb{S}_{1}$ consists of $N_{1} \mathcal{P}$-particles, only the compositions $\zeta_{1}, \zeta_{2}, \ldots, \zeta_{\left(\begin{array}{l}\mathfrak{N} \\ N_{1}\end{array}\right)}$ are admissible for $\mathbb{S}_{1}$. Thus,

$$
\mathcal{H}_{1}:=\bigoplus_{i=1}^{\left(\begin{array}{c}
\mathfrak{N} \\
N_{1}
\end{array}\right)} \mathcal{U}_{1, i}
$$

is an appropriate state space for $\mathbb{S}_{1}$ (cf. Sect. 2.1.1). Correspondingly,

$$
\mathcal{H}_{2}:=\bigoplus_{j=1}^{\left(\begin{array}{c}
\mathfrak{N} \\
N_{2}
\end{array}\right)} \mathcal{U}_{2, j}
$$

is an appropriate state space for $\mathbb{S}_{2}$. Due to Condition 4.5 the tensor product of $\mathcal{H}_{1}$ and $\mathcal{H}_{2}$ does not come into consideration as the state space for the total system $\mathbb{S}_{1+2}$; instead

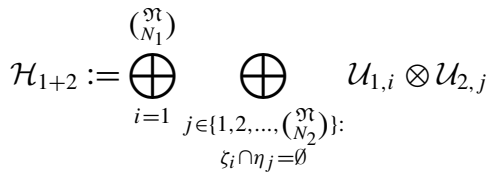

is an appropriate state space for $\mathbb{S}_{1+2}$.

For $\mathrm{m} \in \mathcal{M}_{1}, \mathrm{n} \in \mathcal{M}_{2}$ and $\zeta(\mathrm{m}) \cap \eta(\mathrm{n})=\emptyset,|\mathrm{m}\rangle \otimes|\mathrm{n}\rangle$ is a state vector of $\mathcal{H}_{1+2}$. (In particular, $|\mathrm{m}\rangle \otimes|\mathrm{n}\rangle$ trivially satisfies the symmetrization postulate because the $\mathcal{P}$-particles are pairwise non-identical.) The pure state of $\mathbb{S}_{1+2}$ that is represented by $|m\rangle \otimes|n\rangle$ is referred to as $m \otimes n$.

Remark $4.6 \mathcal{H}_{1+2}$ is not of the form described in Sect. 2.1.1; compared to (2.1) and (2.2), $\mathcal{H}_{1+2}$ has a somewhat more complicated structure, which ensures that in all pure states of $\mathbb{S}_{1+2}$ the subsystems $\mathbb{S}_{1}$ and $\mathbb{S}_{2}$ have the assumed particle numbers $N_{1}$ and $N_{2}$. Alternatively, instead of $\mathcal{H}_{1+2}$,

$$
\mathcal{H}_{1+2}^{\prime}:=\bigoplus_{l=1}^{\substack{\mathfrak{N} \\ N_{1}+N_{2}}} \mathcal{U}_{1+2, l}
$$

may be taken as the state space for $\mathbb{S}_{1+2}$. $\mathcal{H}_{1+2}^{\prime}$ is structured as described in Sect. 2.1.1 and contains $\mathcal{H}_{1+2}$ as a vector subspace. However, in this case, all microstates that are represented by state vectors in $\mathcal{H}_{1+2}^{\prime} \backslash \mathcal{H}_{1+2}$ must possess the probability zero because otherwise $\mathbb{S}_{1}$ and $\mathbb{S}_{2}$ do not have the assumed particle numbers $N_{1}$ and $N_{2}$.

Remark 4.7 Since $\mathbb{S}_{1}$ and $\mathbb{S}_{2}$ are closed with respect to particle exchange, transitions between two pure states of $\mathbb{S}_{1+2}$ that differ with regard to the composition of $\mathbb{S}_{1}$ or $\mathbb{S}_{2}$ are forbidden. In particular, these transitions are still forbidden even if the two pure states belong to the same composition of $\mathbb{S}_{1+2}$ (cf. Sect. 4.2.1).

4.3.2.2 Macrostate of the Total System The total system $\mathbb{S}_{1+2}$ is in the macrostate $\mathbf{M}_{1+2}$ which is defined as follows:

$$
\mathcal{M}_{1+2}=\left\{\mathrm{m} \otimes \mathrm{n}: \mathrm{m} \in \mathcal{M}_{1} \wedge \mathrm{n} \in \mathcal{M}_{2} \wedge \zeta(\mathrm{m}) \cap \eta(\mathrm{n})=\emptyset\right\}
$$


is the set of the microstates of $\mathrm{M}_{1+2}$, and

$$
P_{\mathrm{M}_{1+2}}(\mathrm{~m} \otimes \mathrm{n})=\frac{\mathfrak{N} !\left(\mathfrak{N}-N_{1}-N_{2}\right) !}{\left(\mathfrak{N}-N_{1}\right) !\left(\mathfrak{N}-N_{2}\right) !} P_{\mathrm{M}_{1}}(\mathrm{~m}) P_{\mathrm{M}_{2}}(\mathrm{n})
$$

is the probability of $m \otimes n \in \mathcal{M}_{1+2}$ in $M_{1+2}$.

Proof According to (4.29) and the rules of probability calculus (marginalization), the probability of $m \in \mathcal{M}_{1}$ in $M_{1+2}$ is

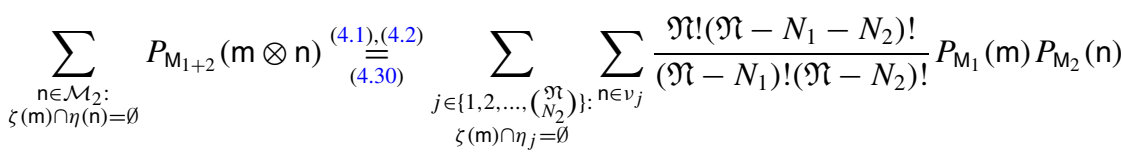

$$
\begin{aligned}
& \stackrel{(4.3)}{=} \frac{\left(\begin{array}{c}
\mathfrak{N} \\
N_{2}
\end{array}\right)}{\left(\begin{array}{c}
\mathfrak{N}-N_{1} \\
N_{2}
\end{array}\right)} P_{\mathrm{M}_{1}}(\mathrm{~m}) \sum_{\substack{j \in\left\{1,2, \ldots,\left(\begin{array}{c}
\mathfrak{N} \\
N_{2}
\end{array}\right)\right\}: \\
\zeta(\mathrm{m}) \cap \eta_{j}=\emptyset}} P_{\mathrm{M}_{2}}\left(\eta_{j}\right) \\
& \stackrel{(4.22)}{=} \quad P_{\mathrm{M}_{1}}(\mathrm{~m})
\end{aligned}
$$

and, correspondingly, the probability of $\mathrm{n} \in \mathcal{M}_{2}$ in $\mathrm{M}_{1+2}$ is $P_{\mathrm{M}_{2}}(\mathrm{n})$. Thus, if the total system $\mathbb{S}_{1+2}$ is in the macrostate $M_{1+2}$, then the two subsystems $\mathbb{S}_{1}$ and $\mathbb{S}_{2}$ are in the macrostates $M_{1}$ and $\mathrm{M}_{2}$ as required.

Further, in $\mathrm{M}_{1+2}$, the conditional probability $P_{\mathrm{M}_{1+2}}(\mathrm{~m} \mid \mathrm{n})$ of the microstate $\mathrm{m} \in \mathcal{M}_{1}$, given that the microstate $\mathrm{n} \in \mathcal{M}_{2}$ is certain, is

$$
P_{\mathrm{M}_{1+2}}(\mathrm{~m} \mid \mathrm{n}) \stackrel{(4.30)}{=} \begin{cases}\frac{\mathfrak{N} !\left(\mathfrak{N}-N_{1}-N_{2}\right) !}{\left(\mathfrak{N}-N_{1}\right) !\left(\mathfrak{N}-N_{2}\right) !} P_{\mathrm{M}_{1}}(\mathrm{~m}) & \text { for } \zeta(\mathrm{m}) \cap \eta(\mathrm{n})=\emptyset \\ 0 & \text { otherwise }\end{cases}
$$

Hence, $P_{\mathrm{M}_{1+2}}(\mathrm{~m} \mid \mathrm{n})$ is the same for all $\mathrm{n} \in \mathcal{M}_{2}$ that are compatible with $\mathrm{m}$ regarding Condition 4.5; an analogous statement holds for the conditional probability $P_{\mathrm{M}_{1+2}}(\mathrm{n} \mid \mathrm{m})$. Thus, in $\mathrm{M}_{1+2}, \mathbb{S}_{1}$ and $\mathbb{S}_{2}$ are correlated only by Condition 4.5 as required.

Furthermore, $\mathrm{M}_{1+2}$ is well-defined: According to Sect. 4.3.2.1, all elements of $\mathcal{M}_{1+2}$ are pure states of $\mathbb{S}_{1+2}$. For $m \otimes n, m^{\prime} \otimes n^{\prime} \in \mathcal{M}_{1+2}$, it holds that

$$
\begin{gathered}
\left\langle\mathrm{m} \otimes \mathrm{n} \mid \mathrm{m}^{\prime} \otimes \mathrm{n}^{\prime}\right\rangle=\left(e ^ { - \mathrm { i } \varphi } \left\langle\mathrm{m}|\otimes\langle\mathrm{n}|)\left(e^{\mathrm{i} \varphi^{\prime}}\left|\mathrm{m}^{\prime}\right\rangle \otimes\left|\mathrm{n}^{\prime}\right\rangle\right)=e^{\mathrm{i}\left(\varphi^{\prime}-\varphi\right)}\left\langle\mathrm{m} \mid \mathrm{m}^{\prime}\right\rangle\left\langle\mathrm{n} \mid \mathrm{n}^{\prime}\right\rangle\right.\right. \\
\stackrel{(2.3)}{=} e^{\mathrm{i}\left(\varphi^{\prime}-\varphi\right)} \delta_{\mathrm{m}, \mathrm{m}^{\prime}} \delta_{\mathrm{n}, \mathrm{n}^{\prime}}=\delta_{\mathrm{m} \otimes \mathrm{n}, \mathrm{m}^{\prime} \otimes \mathrm{n}^{\prime}} \quad\left(\varphi, \varphi^{\prime} \in \mathbb{R}\right)
\end{gathered}
$$

thus, the microstates of $\mathrm{M}_{1+2}$ satisfy the condition (2.3). The condition (2.4) and the normalization condition

$$
\sum_{\mathrm{m} \otimes \mathrm{n} \in \mathcal{M}_{1+2}} P_{\mathrm{M}_{1+2}}(\mathrm{~m} \otimes \mathrm{n}) \stackrel{(4.29),(4.31)}{=} \sum_{\mathrm{m} \in \mathcal{M}_{1}} P_{\mathrm{M}_{1}}(\mathrm{~m}) \stackrel{(2.5)}{=} 1
$$

are also fulfilled for $\mathrm{M}_{1+2}$. 


\subsubsection{3 $\mathcal{P}$-harmonicity of the Total System The total system $\mathbb{S}_{1+2}$ is $\mathcal{P}$-harmonic.}

Proof Let $\xi_{l} \subset \mathcal{M}_{1+2}$ be the set of those microstates of $\mathrm{M}_{1+2}$ that belong to the composition $\theta_{l}$. For each composition $\theta_{l}$ of $\mathbb{S}_{1+2}$ there are $\left(\begin{array}{c}N_{1}+N_{2} \\ N_{1}\end{array}\right)$ decompositions

$$
\begin{aligned}
\left(\zeta_{m_{l, z}}, \eta_{n_{l, z}}\right) ; & z=1,2, \ldots,\left(\begin{array}{c}
N_{1}+N_{2} \\
N_{1}
\end{array}\right), \\
& m_{l, z} \in\left\{1,2, \ldots,\left(\begin{array}{c}
\mathfrak{N} \\
N_{1}
\end{array}\right)\right\}, \quad n_{l, z} \in\left\{1,2, \ldots,\left(\begin{array}{c}
\mathfrak{N} \\
N_{2}
\end{array}\right)\right\}
\end{aligned}
$$

with

$$
\zeta_{m_{l, z}} \cup \eta_{n_{l, z}}=\theta_{l} \quad \text { and } \quad \zeta_{m_{l, z}} \cap \eta_{n_{l, z}}=\emptyset .
$$

Hence, the probability $P_{\mathrm{M}_{1+2}}\left(\theta_{l}\right)$ that $\mathbb{S}_{1+2}$ has the composition $\theta_{l}$ in $\mathrm{M}_{1+2}$ is

$$
\begin{aligned}
& P_{\mathrm{M}_{1+2}}\left(\theta_{l}\right) \quad \stackrel{(4.3)}{=} \quad \sum_{\mathrm{m} \otimes \mathrm{n} \in \xi_{l}} P_{\mathrm{M}_{1+2}}(\mathrm{~m} \otimes \mathrm{n}) \\
& \text { (4.29),(4.35),(4.36) } \sum_{z=1}^{\left(\begin{array}{c}
N_{1}+N_{2} \\
N_{1}
\end{array}\right)} \sum_{\mathrm{m} \in \mu_{m_{l, z}}} \sum_{\mathrm{n} \in v_{n_{l, z}}} P_{\mathrm{M}_{1+2}}(\mathrm{~m} \otimes \mathrm{n}) \\
& \stackrel{(4.30),(4.3)}{=} \sum_{z=1}^{\left(\begin{array}{c}
N_{1}+N_{2} \\
N_{1}
\end{array}\right)} \frac{\mathfrak{N} !\left(\mathfrak{N}-N_{1}-N_{2}\right) !}{\left(\mathfrak{N}-N_{1}\right) !\left(\mathfrak{N}-N_{2}\right) !} P_{\mathrm{M}_{1}}\left(\zeta_{m_{l, z}}\right) P_{\mathrm{M}_{2}}\left(\eta_{n_{l, z}}\right) \\
& \stackrel{(4.22)}{=} \quad\left(\begin{array}{c}
N_{1}+N_{2} \\
N_{1}
\end{array}\right) \frac{\mathfrak{N} !\left(\mathfrak{N}-N_{1}-N_{2}\right) !}{\left(\mathfrak{N}-N_{1}\right) !\left(\mathfrak{N}-N_{2}\right) !} \frac{1}{\left(\begin{array}{c}
\mathfrak{N} \\
N_{1}
\end{array}\right)} \frac{1}{\left(\begin{array}{c}
\mathfrak{N} \\
N_{2}
\end{array}\right)} \\
& =\frac{1}{\left(\begin{array}{c}
\mathfrak{N} \\
N_{1}+N_{2}
\end{array}\right)} \text {. }
\end{aligned}
$$

Thus, $P_{\mathrm{M}_{1+2}}\left(\theta_{l}\right)$ is independent of $l$, and so all compositions of $\mathbb{S}_{1+2}$ consisting of $N_{1}+N_{2}$ $\mathcal{P}$-particles have the same probability $\frac{1}{\left(\frac{\mathfrak{N}}{N_{1}+N_{2}}\right)}$, i.e., $\mathbb{S}_{1+2}$ is $\mathcal{P}$-harmonic.

4.3.2.4 Entropy of the Total System The entropy of the total system $\mathbb{S}_{1+2}$ is

$$
S\left(\mathrm{M}_{1+2}\right)=S\left(\mathrm{M}_{1}\right)+S\left(\mathrm{M}_{2}\right)-k \ln \frac{\mathfrak{N} !\left(\mathfrak{N}-N_{1}-N_{2}\right) !}{\left(\mathfrak{N}-N_{1}\right) !\left(\mathfrak{N}-N_{2}\right) !} .
$$

Proof It holds that

$$
\begin{aligned}
S\left(\mathrm{M}_{1+2}\right) \stackrel{(2.7)}{=}-k \sum_{\mathrm{m} \otimes \mathrm{n} \in \mathcal{M}_{1+2}} P_{\mathrm{M}_{1+2}}(\mathrm{~m} \otimes \mathrm{n}) \ln P_{\mathrm{M}_{1+2}}(\mathrm{~m} \otimes \mathrm{n}) \\
\stackrel{(4.30),(4.29)}{=}-k \sum_{\mathrm{m} \otimes \mathrm{n} \in \mathcal{M}_{1+2}} P_{\mathrm{M}_{1+2}}(\mathrm{~m} \otimes \mathrm{n}) \ln \frac{\mathfrak{N} !\left(\mathfrak{N}-N_{1}-N_{2}\right) !}{\left(\mathfrak{N}-N_{1}\right) !\left(\mathfrak{N}-N_{2}\right) !} \\
-k \sum_{\mathrm{m} \in \mathcal{M}_{1}} \ln P_{\mathrm{M}_{1}}(\mathrm{~m}) \sum_{\substack{\mathrm{n} \in \mathcal{M}_{2}: \\
\zeta(\mathrm{m}) \cap \eta(\mathrm{n})=\emptyset}} P_{\mathrm{M}_{1+2}}(\mathrm{~m} \otimes \mathrm{n})
\end{aligned}
$$




$$
\begin{gathered}
-k \sum_{\mathrm{n} \in \mathcal{M}_{2}} \ln P_{\mathrm{M}_{2}}(\mathrm{n}) \sum_{\substack{\mathrm{m} \in \mathcal{M}_{1}: \\
\zeta(\mathrm{m}) \cap \eta(\mathrm{n})=\emptyset}} P_{\mathrm{M}_{1+2}}(\mathrm{~m} \otimes \mathrm{n}) \\
\stackrel{(2.5),(4.31)}{=}-k \ln \frac{\mathfrak{N} !\left(\mathfrak{N}-N_{1}-N_{2}\right) !}{\left(\mathfrak{N}-N_{1}\right) !\left(\mathfrak{N}-N_{2}\right) !} \\
-k \sum_{\mathrm{m} \in \mathcal{M}_{1}} P_{\mathrm{M}_{1}}(\mathrm{~m}) \ln P_{\mathrm{M}_{1}}(\mathrm{~m})-k \sum_{\mathrm{n} \in \mathcal{M}_{2}} P_{\mathrm{M}_{2}}(\mathrm{n}) \ln P_{\mathrm{M}_{2}}(\mathrm{n}) \\
\stackrel{(2.7)}{=} \quad S\left(\mathrm{M}_{1}\right)+S\left(\mathrm{M}_{2}\right)-k \ln \frac{\mathfrak{N} !\left(\mathfrak{N}-N_{1}-N_{2}\right) !}{\left(\mathfrak{N}-N_{1}\right) !\left(\mathfrak{N}-N_{2}\right) !} .
\end{gathered}
$$

Remark 4.8 The entropy of the total system $\mathbb{S}_{1+2}$ is not additively composed of the entropies of the two subsystems $\mathbb{S}_{1}$ and $\mathbb{S}_{2}$ because $\mathbb{S}_{1}$ and $\mathbb{S}_{2}$ are correlated due to Condition 4.5. Only in the limiting case $\mathfrak{N} \gg N_{1}+N_{2}$ is this correlation negligible and the entropy approximately additive.

\subsubsection{Reduced Entropy}

4.3.3.1 Definition and Properties Consider again the system $\mathbb{S}$ of $N \mathcal{P}$-particles. Let the reduced entropy $R$ of a $\mathcal{P}$-harmonic macrostate $\mathrm{M}$ of $\mathbb{S}$ be defined as

$$
R(\mathrm{M}):=S(\mathrm{M})-k \ln \frac{\mathfrak{N} !}{(\mathfrak{N}-N) !} .
$$

Plugging (4.23) into (4.39) yields

$$
R(\mathrm{M})=\bar{S}(\mathrm{M})-k \ln N !
$$

The reduced entropy (in contrast to the entropy) is additive for $\mathcal{P}$-harmonic systems that are correlated only by Condition 4.5; instead of (4.38), it holds that

$$
\begin{aligned}
R\left(\mathrm{M}_{1+2}\right) & \stackrel{(4.39)}{=} S\left(\mathrm{M}_{1+2}\right)-k \ln \frac{\mathfrak{N} !}{\left(\mathfrak{N}-\left(N_{1}+N_{2}\right)\right) !} \\
& \stackrel{(4.38)}{=} S\left(\mathrm{M}_{1}\right)+S\left(\mathrm{M}_{2}\right)-k \ln \frac{\mathfrak{N} !\left(\mathfrak{N}-N_{1}-N_{2}\right) !}{\left(\mathfrak{N}-N_{1}\right) !\left(\mathfrak{N}-N_{2}\right) !}-k \ln \frac{\mathfrak{N} !}{\left(\mathfrak{N}-N_{1}-N_{2}\right) !} \\
& =S\left(\mathrm{M}_{1}\right)+S\left(\mathrm{M}_{2}\right)-k \ln \frac{\mathfrak{N} !}{\left(\mathfrak{N}-N_{1}\right) !}-k \ln \frac{\mathfrak{N} !}{\left(\mathfrak{N}-N_{2}\right) !} \\
& \stackrel{(4.39)}{=} R\left(\mathrm{M}_{1}\right)+R\left(\mathrm{M}_{2}\right) .
\end{aligned}
$$

Furthermore, the difference $\Delta R$ between the reduced entropies of two $\mathcal{P}$-harmonic macrostates $\mathrm{M}_{\text {before }}$ and $\mathrm{M}_{\text {after }}$ of $\mathbb{S}$ agrees with the entropy difference $\Delta S$ of these macrostates:

$$
\Delta R=R\left(\mathrm{M}_{\mathrm{after}}\right)-R\left(\mathrm{M}_{\text {before }}\right) \stackrel{(4.39)}{=} S\left(\mathrm{M}_{\mathrm{after}}\right)-S\left(\mathrm{M}_{\text {before }}\right)=\Delta S .
$$

[ $\mathfrak{N}$ as well as $N$ are the same for $\mathrm{M}_{\text {before }}$ and $\mathrm{M}_{\text {after }}$ because of particle conservation (see Sect. 2.3) and the closedness of $\mathbb{S}$ with respect to particle exchange.] Physical predictions do 
not depend on the absolute value of the entropy but only on entropy differences. Hence, due to (4.42), the reduced entropy of $\mathcal{P}$-harmonic closed systems is equivalent to the entropy. This equivalence combined with the additivity (4.41) makes the reduced entropy for $\mathcal{P}$ harmonic systems a thermodynamic potential (see Sect. 4.3.3.2).

Remark 4.9 The reduced entropy, expressed by formula (4.40), does not (directly) depend on $\mathfrak{N}$. Thus, under certain circumstances, the reduced entropy (in contrast to the entropy) can be determined even without knowledge of $\mathfrak{N}$.

\subsubsection{Thermodynamic Description of P-harmonic Systems by Means of the Reduced En-}

tropy For a $\mathcal{P}$-harmonic system with fixed particle number, the composition probabilities are stipulated by (4.22); thus, a microcanonical equilibrium state of the system is already completely characterized by the energy $E$, the particle number $N$ and, if any, the values of the alterable system parameters [cf. Theorem 4.2].

As is known, the equilibrium entropy $S$ as a function of its natural variables $E, N, \ldots$ is for a system of indistinguishable particles a thermodynamic potential (in the broader sense), that is, all thermodynamic properties of the system result from the partial derivatives of $S(E, N, \ldots)$. However, as will be shown below, for a $\mathcal{P}$-harmonic system, the partial derivatives of the reduced entropy $R(E, N, \ldots)$ yield the thermodynamic properties of the system. Due to (4.39), $S(E, N, \ldots)$ and $R(E, N, \ldots)$ differ in the partial derivative with respect to the particle number $N$. Hence, for $\mathcal{P}$-harmonic systems, in particular $-\frac{\partial S}{\partial N} / \frac{\partial S}{\partial E}$ relinquishes its thermodynamic interpretation as chemical potential to $-\frac{\partial R}{\partial N} / \frac{\partial R}{\partial E}$.

Because of the energy and particle conservation (see Sect. 2.3), the alteration of the energy $E_{1}$ and the particle number $N_{1}$ of a system $\mathbb{S}_{1}$ always causes a change in the energy $E_{2}$ and the particle number $N_{2}$ of another system $\mathbb{S}_{2}$ absorbing (releasing) the particles and the energy from (to) $\mathbb{S}_{1}$. Let $\mathbb{S}_{2}$ be chosen such that the total system $\mathbb{S}_{1+2}$ consisting of $\mathbb{S}_{1}$ and $\mathbb{S}_{2}$ is isolated. It then holds for the variations of the energies and the particle numbers of $\mathbb{S}_{1}$ and $\mathbb{S}_{2}$

$$
\Delta N_{1}=-\Delta N_{2} \text { and } \Delta E_{1}=-\Delta E_{2} .
$$

According to the second law of thermodynamics, the exchange of energy and particles between $\mathbb{S}_{1}$ and $\mathbb{S}_{2}$ leads to an entropy change

$$
\Delta S_{1+2} \geq 0
$$

in $\mathbb{S}_{1+2}$. Now, let it be assumed that before and after the exchange, $\mathbb{S}_{1}$ and $\mathbb{S}_{2}$ each are in microcanonical equilibrium and are correlated only by Condition 4.5. Further, let it be assumed that before and after the exchange, both systems (and thus, according to Sect. 4.3.2.3, also the total system $\left.\mathbb{S}_{1+2}\right)$ are $\mathcal{P}$-harmonic. Let $S_{1}\left(E_{1}, N_{1}, \ldots\right)$ and $S_{2}\left(E_{2}, N_{2}, \ldots\right)$ be the entropies, and $R_{1}\left(E_{1}, N_{1}, \ldots\right)$ and $R_{2}\left(E_{2}, N_{2}, \ldots\right)$ the reduced entropies of $\mathbb{S}_{1}$ and $\mathbb{S}_{2}$. For small changes of the energy and the particle number, $\Delta S_{1+2}$ can be expressed by the partial derivatives of $R_{1}$ and $R_{2}$ as follows:

$$
\begin{aligned}
\Delta S_{1+2} & \stackrel{(4.42)}{=} \Delta R_{1+2} \stackrel{(4.41)}{=} \Delta R_{1}+\Delta R_{2} \\
& \approx \frac{\partial R_{1}}{\partial E_{1}} \Delta E_{1}+\frac{\partial R_{1}}{\partial N_{1}} \Delta N_{1}+\frac{\partial R_{2}}{\partial E_{2}} \Delta E_{2}+\frac{\partial R_{2}}{\partial N_{2}} \Delta N_{2} .
\end{aligned}
$$


This relation turns the reduced entropy into a thermodynamic potential. As usual, one identifies $T_{1}:=1 / \frac{\partial R_{1}}{\partial E_{1}}$ with the temperature and $\mu_{1}:=-T_{1} \frac{\partial R_{1}}{\partial N_{1}}$ with the chemical potential of $\mathbb{S}_{1}$ (correspondingly, for $\mathbb{S}_{2}, T_{2}:=1 / \frac{\partial R_{2}}{\partial E_{2}}$ and $\mu_{2}:=-T_{2} \frac{\partial R_{2}}{\partial N_{2}}$ ). With this, one obtains

$$
\begin{gathered}
\Delta S_{1+2} \stackrel{\stackrel{(4.45)}{=}}{=} \frac{1}{T_{1}} \Delta E_{1}-\frac{\mu_{1}}{T_{1}} \Delta N_{1}+\frac{1}{T_{2}} \Delta E_{2}-\frac{\mu_{2}}{T_{2}} \Delta N_{2} \\
\stackrel{(4.43)}{=}\left(\frac{1}{T_{1}}-\frac{1}{T_{2}}\right) \Delta E_{1}-\left(\frac{\mu_{1}}{T_{1}}-\frac{\mu_{2}}{T_{2}}\right) \Delta N_{1} .
\end{gathered}
$$

If, at first, $\mathbb{S}_{1}$ and $\mathbb{S}_{2}$ can only exchange energy but no particles, then it holds that $\Delta N_{1}=0$. From (4.46) it then follows due to (4.44) that energy flows from the system with higher temperature to the system with lower temperature. If, after $\mathbb{S}_{1}$ and $\mathbb{S}_{2}$ have reached thermal equilibrium $\left(T_{1}=T_{2}\right)$, one enables in addition to the energy exchange an exchange of particles between the two systems, then it correspondingly follows that the system with higher chemical potential loses particles to the system with lower chemical potential.

Because, according to (4.38), a particle exchange between $\mathbb{S}_{1}$ and $\mathbb{S}_{2}$ changes the correlation between the two systems, $\Delta S_{1+2}$ is not the sum of the entropy changes that $\mathbb{S}_{1}$ and $\mathbb{S}_{2}$ each individually experience through the particle exchange. Thus, $\Delta S_{1+2}$ cannot be expressed analogously to (4.45) by the partial derivatives of $S_{1}$ and $S_{2}$. For that reason, $-T_{1} \frac{\partial S_{1}}{\partial N_{1}}$ and $-T_{2} \frac{\partial S_{2}}{\partial N_{2}}$ lose for $\mathbb{S}_{1}$ and $\mathbb{S}_{2}$ the thermodynamic interpretation as chemical potential; instead, as stated above, $-T_{1} \frac{\partial R_{1}}{\partial N_{1}}$ and $-T_{2} \frac{\partial R_{2}}{\partial N_{2}}$ assume this role. To summarize:

Theorem 4.10 For harmonic systems of non-identical particles, no longer the entropy, but the reduced entropy as a function of its natural variables $E, N, \ldots$ is a thermodynamic potential.

Other thermodynamic potentials have to be replaced in an analogous manner; for example, the free energy by the reduced free energy $E(T, N, \ldots)-T R(T, N, \ldots)$.

Remark 4.11 Cheng in [6] also defines a "reduced entropy" for classical systems of distinguishable particles. However, his definition is based on an entropy that does not agree with (4.16).

Remark 4.12 Swendsen in [39] proposes a new, unfortunately somewhat imprecise definition of entropy. At least for a classical ideal gas of distinguishable particles, the entropy defined by Swendsen agrees with the reduced entropy. Swendsen's redefinition of entropy is criticized by Nagle in [29]; Nagle shows by means of several thought experiments that Swendsen's redefinition fails for non-harmonic systems. Swendsen's response [40] to this criticism suggests that Swendsen in [39] implicitly assumed harmonic systems.

Therefore, at this point, the reader is reminded that the scope of application of the reduced entropy is limited to harmonic systems and that harmonic systems still have an entropy (differing from the reduced entropy).

\section{Statistics of Distinguishable Identical Classical Particles}

Let a set $\mathcal{R}$ of $\mathfrak{N}$ pairwise distinguishable identical classical particles be given. Let every $\mathcal{R}$-particle be non-identical to every particle not belonging to $\mathcal{R}$. Further, let a system $\mathbb{S}$ 
of $N(N \leq \mathfrak{N}) \mathcal{R}$-particles be given that is closed with respect to particle exchange. Let $\zeta_{1}, \zeta_{2}, \ldots, \zeta_{\left(\begin{array}{l}\mathfrak{N} \\ N\end{array}\right)}$ be the compositions of $\mathbb{S}$ consisting of $N \mathcal{R}$-particles and $\mathcal{U}_{i}$ the phase space spanned by the generalized coordinates and momenta of the $\zeta_{i}$-particles. As reasoned in Sect. 4.1.2,

$$
\mathcal{H}:=\bigcup_{i=1}^{\left(\begin{array}{c}
\mathfrak{N} \\
N
\end{array}\right)} \mathcal{U}_{i}
$$

can be taken as the set of all phase space points of $\mathbb{S}$. For simplicity, it is assumed in the following that the $\mathcal{R}$-particles have no internal degrees of freedom and thus, the phase spaces $\mathcal{U}_{1}, \mathcal{U}_{2}, \ldots, \mathcal{U}_{\left(\begin{array}{l}\mathfrak{N} \\ N\end{array}\right)}$ are all $6 N$-dimensional.

\subsection{Postulate for Distinguishable Identical Classical Particles}

For a given point in time, let the positions and conjugate momenta of the particles in $\mathbb{S}$ be known. Since the $\mathcal{R}$-particles are identical, it cannot in principal be known which $N$ of the $\mathfrak{N} \mathcal{R}$-particles are located in $\mathbb{S}$ or by which $\mathcal{R}$-particle a particular conjugate positionmomentum pair is occupied. Since no occupation alternative should be favored over the others, it is reasonable to consider all occupation possibilities equally:

Postulate 5.1 In every macrostate, phase space points arising from each other through permutation of distinguishable identical classical particles possess the same probability density.

\subsection{Harmonicity}

Let $\mathbb{S}$ be in the macrostate M. $\left\langle\zeta_{i} ; \vec{q}, \vec{p}\right\rangle$ denotes the point in $\mathcal{U}_{i}$ with the coordinates $\vec{q}, \vec{p}$. For the composition probabilities of $\mathbb{S}$, it holds that

$$
\begin{gathered}
P_{\mathrm{M}}\left(\zeta_{i}\right) \stackrel{(4.11)}{=} \int \mathrm{d} \mathcal{U}_{i} \rho_{\mathrm{M}}(\widehat{\mathrm{m}})=\int \mathrm{d}^{3 N} q \mathrm{~d}^{3 N} p \rho_{\mathrm{M}}\left(\left\langle\zeta_{i} ; \vec{q}, \vec{p}\right\rangle\right) \\
\stackrel{\text { Postulate }}{=} \int .1 \\
\stackrel{(4.11)}{=} \quad \mathrm{d}^{3 N} q \mathrm{~d}^{3 N} p \rho_{\mathrm{M}}\left(\left\langle\zeta_{j} ; \vec{q}, \vec{p}\right\rangle\right)=\int \mathrm{d} \mathcal{U}_{j} \rho_{\mathrm{M}}(\widehat{\mathrm{m}}) \\
\left.P_{j}\right) \quad \forall i, j \in\left\{1,2, \ldots,\left(\begin{array}{c}
\mathfrak{N} \\
N
\end{array}\right)\right\}
\end{gathered}
$$

and thus

$$
P_{\mathrm{M}}\left(\zeta_{1}\right)=P_{\mathrm{M}}\left(\zeta_{2}\right)=\cdots=P_{\mathrm{M}}\left(\zeta_{\left(\begin{array}{c}
\mathfrak{N} \\
N
\end{array}\right)} \stackrel{(4.12)}{=} \frac{1}{\left(\begin{array}{l}
\mathfrak{N} \\
N
\end{array}\right)},\right.
$$

i.e., $M$ is $\mathcal{R}$-harmonic. The generalization to systems whose particle number is not fixed is unproblematic and yields:

Theorem 5.2 A system of distinguishable identical classical particles of the kind $\mathcal{R}$ is always $\mathcal{R}$-harmonic. 


\subsection{Minimal State}

\subsubsection{Definition}

Let $\mathrm{M}$ be a macrostate of $\mathbb{S}$. For the moment, let it be assumed that the underlying particle set $\mathcal{R}$ of $\mathbb{S}$ consists only of $N$ (instead of $\mathfrak{N}$ ) particles. (Thus, the size of $\mathcal{R}$ is assumed minimal; if $\mathcal{R}$ would consist of less particles, then the particle number of $\mathbb{S}$ could no longer be $N$.) Without loss of generality, let $\mathcal{R}$ consist of the particles of the composition $\zeta_{1}$. Then, instead of (5.1),

$$
\underline{\mathcal{H}}:=\mathcal{U}_{1}
$$

can be taken as the set of all phase space points of $\mathbb{S}$.

Let the minimal state $\underline{M}$ associated with $\mathrm{M}$ (which refers to an underlying particle set of $\mathfrak{N}$ particles) be defined through

$$
\rho_{\underline{M}}(\widehat{\mathrm{m}}):=\rho_{\mathrm{M}}\left(\widehat{\mathrm{m}} \mid \zeta_{1}\right) \quad \forall \widehat{\mathrm{m}} \in \underline{\mathcal{H}} .
$$

It holds that

$$
\rho_{\underline{\mathrm{M}}}(\widehat{\mathrm{m}}) \stackrel{(5.5)}{=} \rho_{\mathrm{M}}\left(\widehat{\mathrm{m}} \mid \zeta_{1}\right) \stackrel{(4.13),(5.4)}{=} \frac{\rho_{\mathrm{M}}(\widehat{\mathrm{m}})}{P_{\mathrm{M}}\left(\zeta_{1}\right)} \stackrel{(5.3)}{=}\left(\begin{array}{c}
\mathfrak{N} \\
N
\end{array}\right) \rho_{\mathrm{M}}(\widehat{\mathrm{m}}) \quad \forall \widehat{\mathrm{m}} \in \underline{\mathcal{H}} .
$$

$\underline{M}$ is well-defined by (5.5): $\underline{M}$ satisfies the normalization condition

$$
\int \mathrm{d} \underline{\mathcal{H}} \rho_{\underline{\mathrm{M}}}(\widehat{\mathrm{m}}) \stackrel{(5.4),(5.5)}{=} \int \mathrm{d} \mathcal{U}_{1} \rho_{\mathrm{M}}\left(\widehat{\mathrm{m}} \mid \zeta_{1}\right) \stackrel{(4.14)}{=} 1
$$

and further complies with Postulate 5.1, because, if $\widehat{\mathrm{m}} \in \underline{\mathcal{H}}$ and $\widehat{\mathrm{n}} \in \underline{\mathcal{H}}$ arise from each other through permutation of $\mathcal{R}$-particles, then it holds that

$$
\rho_{\underline{\mathrm{M}}}(\widehat{\mathrm{m}}) \stackrel{(5.6)}{=}\left(\begin{array}{l}
\mathfrak{N} \\
N
\end{array}\right) \rho_{\mathrm{M}}(\widehat{\mathrm{m}}) \stackrel{\text { Postulate }}{=} 5.1\left(\begin{array}{l}
\mathfrak{N} \\
N
\end{array}\right) \rho_{\mathrm{M}}(\widehat{\mathrm{n}}) \stackrel{(5.6)}{=} \rho_{\underline{\mathrm{M}}}(\widehat{\mathrm{n}}) \text {. }
$$

\subsubsection{Entropy of a Macrostate}

Now, let $\mathcal{R}$ consist of all $\mathfrak{N}$ particles again. For each macrostate $\mathrm{M}$ of $\mathbb{S}$ it holds that

$$
\begin{aligned}
& \rho_{\mathrm{M}}\left(\left\langle\zeta_{i} ; \vec{q}, \vec{p}\right\rangle \mid \zeta_{i}\right) \stackrel{(4.13)}{=} \quad \frac{\rho_{\mathrm{M}}\left(\left\langle\zeta_{i} ; \vec{q}, \vec{p}\right\rangle\right)}{P_{\mathrm{M}}\left(\zeta_{i}\right)} \stackrel{(5.3)}{=}\left(\begin{array}{c}
\mathfrak{N} \\
N
\end{array}\right) \rho_{\mathrm{M}}\left(\left\langle\zeta_{i} ; \vec{q}, \vec{p}\right\rangle\right) \\
& \stackrel{\text { Postulate } 5.1}{=}\left(\begin{array}{l}
\mathfrak{N} \\
N
\end{array}\right) \rho_{\mathrm{M}}\left(\left\langle\zeta_{1} ; \vec{q}, \vec{p}\right\rangle\right) \\
& \stackrel{(5.4),(5.6)}{=} \rho_{\underline{\mathrm{M}}}\left(\left\langle\zeta_{1} ; \vec{q}, \vec{p}\right\rangle\right) \quad \forall i \in\left\{1,2, \ldots,\left(\begin{array}{c}
\mathfrak{N} \\
N
\end{array}\right)\right\} .
\end{aligned}
$$

From this it follows that

$$
\begin{gathered}
S\left(\mathrm{M} \mid \zeta_{i}\right) \stackrel{(4.15)}{=}-k \int \mathrm{d}^{3 N} q \mathrm{~d}^{3 N} p \rho_{\mathrm{M}}\left(\left\langle\zeta_{i} ; \vec{q}, \vec{p}\right\rangle \mid \zeta_{i}\right) \ln \left[\rho_{\mathrm{M}}\left(\left\langle\zeta_{i} ; \vec{q}, \vec{p}\right\rangle \mid \zeta_{i}\right)(2 \pi \hbar)^{3 N}\right] \\
\stackrel{(5.9)}{=}-k \int \mathrm{d}^{3 N} q \mathrm{~d}^{3 N} p \rho_{\underline{\mathrm{M}}}\left(\left\langle\zeta_{1} ; \vec{q}, \vec{p}\right\rangle\right) \ln \left[\rho_{\underline{\mathrm{M}}}\left(\left\langle\zeta_{1} ; \vec{q}, \vec{p}\right\rangle\right)(2 \pi \hbar)^{3 N}\right]
\end{gathered}
$$




$$
\stackrel{(5.4),(2.11)}{=} S(\underline{\mathrm{M}}) \quad \forall i \in\left\{1,2, \ldots,\left(\begin{array}{c}
\mathfrak{N} \\
N
\end{array}\right)\right\}
$$

and thus

$$
\bar{S}(\mathrm{M}) \stackrel{(4.24)}{=} \frac{1}{\left(\begin{array}{l}
\mathfrak{N} \\
N
\end{array}\right)} \sum_{i=1}^{\left(\begin{array}{c}
\mathfrak{N} \\
N
\end{array}\right)} S\left(\mathrm{M} \mid \zeta_{i}\right) \stackrel{(5.10)}{=} S(\underline{\mathrm{M}}) .
$$

Hence, one obtains for the entropy of $M$

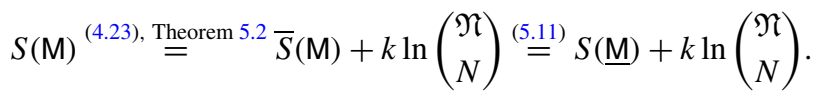

\subsubsection{Equilibrium State}

Let $\mathbb{S}$ be in the microcanonical equilibrium state $\mathrm{Q}$ with the energy $E$. Let $\mu_{i}^{*}$ be the set of all microstates of $\mathbb{S}$ that belong to $\zeta_{i}$ and are a priori possible in $\mathrm{Q}$ (cf. Sect. 4.2). Let $\widehat{\mu_{i}^{*}} \subset \mathcal{U}_{i}$ be the set of phase space points that represent the microstates of $\mu_{i}^{*}$, and

$$
\left\|\widehat{\mu_{i}^{*}}\right\|:=\int_{\widehat{\mu_{i}^{*}}} \mathrm{~d} \mathcal{U}_{i}
$$

the phase space volume of $\widehat{\mu_{i}^{*}}$. As reasoned in Sect. 4.2.1, according to the fundamental postulate (in restricted form), all microstates of $\mu_{i}^{*}$ are equiprobable. Thus, it holds analogously to (4.17) that

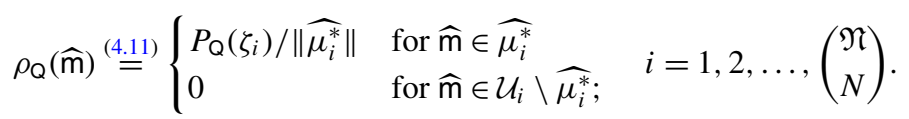

For the moment, let it be assumed again that $\mathcal{R}$ consists only of $N$ (instead of $\mathfrak{N}$ ) particles. Without loss of generality, let $\mathcal{R}$, as in Sect. 5.3.1, consist of the particles of the composition $\zeta_{1}$ such that again $\underline{\mathcal{H}}$ [cf. definition (5.4)] can be taken as the set of all phase space points of $\mathbb{S}$. Apart from that, let $\mathbb{S}$ be unaltered in the microcanonical equilibrium with the energy $E$ (equilibrium state $\mathrm{Q}^{\prime}$ ).

Since all microstates of $\mathbb{S}$ belong to the same composition $\zeta_{1}$, microstates that are a priori possible in $Q^{\prime}$ can generally pass into one another; thus, according to the fundamental postulate, all microstates that are a priori possible in $Q^{\prime}$ are equiprobable. Due to (5.4), the set of microstates that are a priori possible in $\mathrm{Q}^{\prime}$ agrees with $\mu_{1}^{*}$; thus, because of the normalization condition (2.10), it holds that

$$
\rho_{Q^{\prime}}(\widehat{\mathrm{m}})= \begin{cases}1 /\left\|\widehat{\mu_{1}^{*}}\right\| & \text { for } \widehat{\mathrm{m}} \in \widehat{\mu_{1}^{*}} \\ 0 & \text { for } \widehat{\mathrm{m}} \in \underline{\mathcal{H}} \backslash \widehat{\mu_{1}^{*}} .\end{cases}
$$

Comparison of (5.15) with (5.14) shows that

$$
\rho_{\mathrm{Q}^{\prime}}(\widehat{\mathrm{m}}) \stackrel{(5.4)}{=} \frac{\rho_{\mathrm{Q}}(\widehat{\mathrm{m}})}{P_{\mathrm{Q}}\left(\zeta_{1}\right)} \stackrel{(5.3)}{=}\left(\begin{array}{c}
\mathfrak{N} \\
N
\end{array}\right) \rho_{\mathrm{Q}}(\widehat{\mathrm{m}}) \quad \forall \widehat{\mathrm{m}} \in \underline{\mathcal{H}}
$$

and hence

$$
\rho_{\underline{\mathrm{Q}}}(\widehat{\mathrm{m}}) \stackrel{(5.6)}{=}\left(\begin{array}{l}
\mathfrak{N} \\
N
\end{array}\right) \rho_{\mathrm{Q}}(\widehat{\mathrm{m}}) \stackrel{(5.16)}{=} \rho_{\mathrm{Q}^{\prime}}(\widehat{\mathrm{m}}) \quad \forall \widehat{\mathrm{m}} \in \underline{\mathcal{H}} .
$$


Thus, it holds that:

Theorem 5.3 The minimal state $\underline{Q}$ associated with a microcanonical equilibrium state $\mathrm{Q}$ of $\mathbb{S}$ is identical to that microcanonical equilibrium state of $\mathbb{S}$ that agrees with $\mathbf{Q}$ regarding the energy and the values of the alterable system parameters, but for which an underlying particle set $\mathcal{R}$ of only $N$ (instead of $\mathfrak{N}$ ) particles is assumed.

Theorem 5.3, derived for an isolated system with fixed energy, also holds in the canonical case (if "energy" is replaced by "temperature"). The proof of this is similar to the microcanonical case and is therefore not repeated here.

\subsection{Entropy of a Composite System}

Now, let two systems $\mathbb{S}_{1}$ and $\mathbb{S}_{2}$ of $N_{1}$ and $N_{2}\left(N_{1}+N_{2} \leq \mathfrak{N}\right) \mathcal{R}$-particles be given. Let $\mathbb{S}_{1}$ be in the macrostate $M_{1}$ and $\mathbb{S}_{2}$ in the macrostate $M_{2}$. If, apart from Condition 4.5, there is no further correlation between $\mathbb{S}_{1}$ and $\mathbb{S}_{2}$, then, as for harmonic systems of non-identical particles (Sect. 4.3.2.4), it holds for the entropy of the total system consisting of $\mathbb{S}_{1}$ and $\mathbb{S}_{2}$ that

$$
S\left(\mathrm{M}_{1+2}\right)=S\left(\mathrm{M}_{1}\right)+S\left(\mathrm{M}_{2}\right)-k \ln \frac{\mathfrak{N} !\left(\mathfrak{N}-N_{1}-N_{2}\right) !}{\left(\mathfrak{N}-N_{1}\right) !\left(\mathfrak{N}-N_{2}\right) !} .
$$

The proof of (5.18) is similar to the proof of (4.38); Theorem 5.2 guarantees the harmonicity of $\mathrm{M}_{1}$ and $\mathrm{M}_{2}$ required for the proof.

\subsection{Reduced Entropy}

Consider again the system $\mathbb{S}$ of $N \mathcal{R}$-particles. The reduced entropy $R$ of a macrostate $\mathrm{M}$ of $\mathbb{S}$ is defined as for harmonic macrostates of systems of non-identical particles through (4.39). Plugging (5.12) into (4.39) then yields

$$
R(\mathrm{M})=S(\underline{\mathrm{M}})-k \ln N !
$$

The reduced entropy has the properties given in Sect. 4.3.3.1 and therefore replaces the entropy as thermodynamic potential also for systems of distinguishable identical classical particles [cf. Theorem 4.10].

5.6 Equivalence Between Distinguishability and Indistinguishability of Identical Classical Particles

\subsubsection{Partitioning of the Phase Space Points of $\mathbb{S}$ into Classes}

Let the equivalence relation $\sim$ be defined on the set $\mathcal{H}$ of all phase space points of $\mathbb{S}$ through:

Definition 5.4 For $\widehat{m}, \widehat{n} \in \mathcal{H}$, it holds that $\widehat{m} \sim \widehat{n}$ if and only if $\widehat{m}$ and $\widehat{n}$ arise from each other through permutation of $\mathcal{R}$-particles.

The relation $\sim$ partitions $\mathcal{H}$ into equivalence classes; the equivalence class of $\widehat{\mathrm{m}} \in \mathcal{H}$ is

$$
[\widehat{\mathrm{m}}]:=\{\widehat{\mathrm{n}} \in \mathcal{H}: \widehat{\mathrm{n}} \sim \widehat{\mathrm{m}}\} .
$$


The elements of $[\widehat{\mathrm{m}}]$ are called representatives of $[\widehat{\mathrm{m}}]$. Further, let

$$
\mathcal{H} / \sim:=\{[\widehat{\mathrm{m}}]: \widehat{\mathrm{m}} \in \mathcal{H}\}
$$

be the quotient set of $\mathcal{H}$ by $\sim$.

Let $\pi_{1}, \pi_{2}, \ldots, \pi_{N \text { ! }}$ be the elements of the symmetric group of degree $N$. Further, let for a $3 N$-dimensional vector $\vec{q}=\left(q_{1}, q_{2}, \ldots, q_{3 N}\right)$ the three-dimensional vector $\mathbf{q}_{n}$ be defined as

$$
\mathbf{q}_{n}:=\left(q_{3 n-2}, q_{3 n-1}, q_{3 n}\right) ; \quad n=1,2, \ldots, N .
$$

(Thus, if $\vec{q}$ is composed of the generalized coordinates of $N$ particular $\mathcal{R}$-particles, then $\mathbf{q}_{n}$ consists of the generalized coordinates of the $n$th of these $\mathcal{R}$-particles.) The subsets

$$
\begin{aligned}
\widehat{\mu_{i, j}} & :=\left\{\left\langle\zeta_{i} ; \vec{q}, \vec{p}\right\rangle \in \mathcal{H}:\left|\mathbf{q}_{\pi_{j}(1)}\right|<\left|\mathbf{q}_{\pi_{j}(2)}\right|<\cdots<\left|\mathbf{q}_{\pi_{j}(N)}\right|\right\} ; \\
i & =1,2, \ldots,\left(\begin{array}{c}
\mathfrak{N} \\
N
\end{array}\right), \quad j=1,2, \ldots, N !
\end{aligned}
$$

of $\mathcal{H}$ are pairwise disjoint:

$$
\widehat{\mu_{i, j}} \cap \widehat{\mu_{r, s}}=\emptyset \quad \text { for } i \neq r \text { or } j \neq s .
$$

Furthermore, for every two sets $\widehat{\mu_{i, j}}$ and $\widehat{\mu_{r, s}}$ there is a permutation $\sigma_{i, j, r, s}$ of $\mathcal{R}$-particles bijectively mapping $\widehat{\mu_{r, s}}$ to $\widehat{\mu_{i, j}}$ :

$$
\left\{\sigma_{i, j, r, s}(\widehat{\mathrm{m}}): \widehat{\mathrm{m}} \in \widehat{\mu_{r, s}}\right\}=\widehat{\mu_{i, j}} .
$$

Each equivalence class $[\widehat{\mathrm{m}}] \in \mathcal{H} / \sim$ possesses in each of the sets $\widehat{\mu_{i, j}}$ at most one representative. If $[\widehat{\mathrm{m}}]$ possesses in one of the sets $\widehat{\mu_{i, j}}$ a representative, then, due to (5.25), [ $\left.\widehat{\mathrm{m}}\right]$ possesses in each of the sets $\widehat{\mu_{i, j}}$ (exactly) one representative. In addition, the set

$$
\begin{aligned}
\mathcal{H} \backslash \bigcup_{i=1}^{\left(\begin{array}{l}
\mathfrak{N} \\
N
\end{array}\right)} \bigcup_{j=1}^{N !} \widehat{\mu_{i, j}}= & \left\{\left\langle\zeta_{i} ; \vec{q}, \vec{p}\right\rangle \in \mathcal{H}: i \in\left\{1,2, \ldots,\left(\begin{array}{c}
\mathfrak{N} \\
N
\end{array}\right)\right\}\right. \\
& \left.\wedge \exists n, m\left(n, m \in\{1,2, \ldots, N\} \wedge n \neq m \wedge\left|\mathbf{q}_{n}\right|=\left|\mathbf{q}_{m}\right|\right)\right\}
\end{aligned}
$$

of those phase space points of $\mathbb{S}$ that are contained in none of the sets $\widehat{\mu_{i, j}}$ has the phase space volume zero:

$$
\left\|\mathcal{H} \backslash \bigcup_{i=1}^{\left(\begin{array}{l}
\mathfrak{N} \\
N
\end{array}\right)\left(\bigcup_{j=1}^{N !} \widehat{\mu_{i, j}}\right.}\right\|=0 .
$$

Thus, almost every equivalence class $[\widehat{\mathrm{m}}]$ possesses in each of the sets $\widehat{\mu_{i, j}}$ exactly one representative, i.e.,

$$
\left|[\widehat{\mathrm{m}}] \cap \widehat{\mu_{i, j}}\right|=1 \quad \text { for almost all }[\widehat{\mathrm{m}}] \in \mathcal{H} / \sim
$$


and therefore contains $\frac{\mathfrak{N} !}{(\mathfrak{N}-N) !}$ phase space points:

$$
\begin{aligned}
& |[\widehat{\mathrm{m}}]| \stackrel{(5.20)}{=}|[\widehat{\mathrm{m}}] \cap \mathcal{H}| \stackrel{(5.27)}{=}\left|[\widehat{\mathrm{m}}] \cap\left(\bigcup_{i=1}^{\left(\begin{array}{c}
\mathfrak{N} \\
N
\end{array}\right)^{N !}} \bigcup_{j=1}^{\widehat{\mu_{i, j}}}\right)\right|=\left|\bigcup_{i=1}^{\left(\begin{array}{c}
\mathfrak{N} \\
N
\end{array}\right)^{N !}} \bigcup_{j=1}^{N !}\left([\widehat{\mathrm{m}}] \cap \widehat{\mu_{i, j}}\right)\right| \\
& \stackrel{(5.24)}{=} \sum_{i=1}^{\left(\begin{array}{l}
\mathfrak{N} \\
N
\end{array}\right)} \sum_{j=1}^{N !}\left|[\widehat{\mathrm{m}}] \cap \widehat{\mu_{i, j}}\right| \stackrel{(5.28)}{=} \frac{\mathfrak{N} !}{(\mathfrak{N}-N) !} \quad \text { for almost all }[\widehat{\mathrm{m}}] \in \mathcal{H} / \sim \text {. }
\end{aligned}
$$

\subsubsection{Transition to Indistinguishability}

The $\mathcal{R}$-particles can be made indistinguishable by identifying with one another microstates arising from each other through permutation of $\mathcal{R}$-particles. All microstates that are represented by the phase space points of the same equivalence class [ $\widehat{m}]$ thereby merge to one single microstate $\tilde{m}$ represented by each $\widehat{n} \in[\widehat{m}]$. A macrostate $M$ passes through this merging into the macrostate $\widetilde{M}$, in which $\widehat{m}$ possesses the accumulated probability density

$$
\rho_{\widetilde{\mathrm{M}}}(\widehat{\mathrm{m}}):=\sum_{\widehat{\mathrm{n}} \in[\widehat{\mathrm{m}}]} \rho_{\mathrm{M}}(\widehat{\mathrm{n}}) \stackrel{\text { Postulate }}{=} 5.1|[\widehat{\mathrm{m}}]| \rho_{\mathrm{M}}(\widehat{\mathrm{m}}) .
$$

Of course, phase space points representing the same microstate possess the same probability density in $\tilde{\mathrm{M}}$ :

$$
\widehat{\mathrm{m}} \sim \widehat{\mathrm{n}} \Rightarrow \rho_{\widetilde{\mathrm{M}}}(\widehat{\mathrm{m}}) \stackrel{(5.30)}{=}|[\widehat{\mathrm{m}}]| \rho_{\mathrm{M}}(\widehat{\mathrm{m}}) \stackrel{\text { Postulate }}{=} 5.1|[\widehat{\mathrm{n}}]| \rho_{\mathrm{M}}(\widehat{\mathrm{n}}) \stackrel{(5.30)}{=} \rho_{\widetilde{\mathrm{M}}}(\widehat{\mathrm{n}})
$$

Further, $\widetilde{M}$ satisfies the normalization condition (5.40).

In the following, the $\mathcal{R}$-particles, made indistinguishable in the way just described, are denoted $\widetilde{\mathcal{R}}$-particles.

\subsubsection{Measurement of Mechanical Quantities}

For distinguishable $\mathcal{R}$-particles, a mechanical quantity $\mathrm{Q}$ takes on a particular value $\mathrm{Q}(\mathrm{m}, t)$ in every microstate $\mathrm{m}$ and at each point in time $t$. Q can be represented by a function $\widehat{Q}$ of $\widehat{m}$ and $t$ :

$$
\mathrm{Q}(\mathrm{m}, t):=\widehat{\mathrm{Q}}(\widehat{\mathrm{m}}, t) .
$$

The function $\widehat{Q}$ may contain the values of the inner attributes of the $\mathcal{R}$-particles as parameters. For a given $\zeta_{i}, \widehat{Q}\left(\left\langle\zeta_{i} ; \vec{q}, \vec{p}\right\rangle, t\right)$ is a function $\widehat{Q}_{\zeta_{i}}$ of $\vec{q}, \vec{p}$ and $t$ that may contain as parameters only the values of the inner attributes of the $\zeta_{i}$-particles, "inner $\zeta_{i}$-particle values" for short. From $\widehat{Q}_{\zeta_{i}}$ one obtains the function $\widehat{Q}_{\zeta_{j}}$ by replacing the inner $\zeta_{i}$-particle values that appear in $\widehat{Q}_{\zeta_{i}}$ as parameters by the corresponding inner $\zeta_{j}$-particle values. Since all $\mathcal{R}$ particles are identical and thus agree in the values of their inner attributes, it holds that

$$
\begin{aligned}
\widehat{\mathrm{Q}}\left(\left\langle\zeta_{i} ; \vec{q}, \vec{p}\right\rangle, t\right) & =\widehat{\mathrm{Q}}_{\zeta_{i}}(\vec{q}, \vec{p}, t)=\widehat{\mathrm{Q}}_{\zeta_{j}}(\vec{q}, \vec{p}, t) \\
& =\widehat{\mathrm{Q}}\left(\left\langle\zeta_{j} ; \vec{q}, \vec{p}\right\rangle, t\right) \quad \forall i, j \in\left\{1,2, \ldots,\left(\begin{array}{c}
\mathfrak{N} \\
N
\end{array}\right)\right\} .
\end{aligned}
$$


Of course, $Q(\mathrm{~m}, t)$ does not depend on which axes of the phase space are assigned to which particles. Therefore, $\widehat{Q}\left(\left\langle\zeta_{i} ; \vec{q}, \vec{p}\right\rangle, t\right)$ does not change if one transposes two $\zeta_{i}$-particles $\mathfrak{p}_{n_{i, q}}$ and $\mathfrak{p}_{n_{i, r}}$ and at the same time interchanges the values of their inner attributes appearing in $\widehat{Q}$ as parameters. Since $\mathfrak{p}_{n_{i, q}}$ and $\mathfrak{p}_{n_{i, r}}$ are identical, the interchange of the values of their inner attributes is without effect, and so $\widehat{Q}\left(\left\langle\zeta_{i} ; \vec{q}, \vec{p}\right\rangle, t\right)$ is invariant under transposition of $\mathfrak{p}_{n_{i, q}}$ and $\mathfrak{p}_{n_{i, r}}$. The transposition of a $\zeta_{i}$-particle with an identical non- $\zeta_{i}$-particle also does not change $\widehat{Q}\left(\left\langle\zeta_{i} ; \vec{q}, \vec{p}\right\rangle, t\right)$ because, for a suitable assignment of phase space axes to particles, the phase space coordinates $\vec{q}, \vec{p}$ remain unaltered under this transposition, and so the invariance immediately follows from (5.33). Finally, the invariance of $\widehat{Q}\left(\left\langle\zeta_{i} ; \vec{q}, \vec{p}\right\rangle, t\right)$ under transposition of two non- $\zeta_{i}$-particles is trivial. Hence, $\widehat{Q}$ is invariant under permutation of any $\mathcal{R}$-particles:

$$
\widehat{\mathrm{m}} \sim \widehat{\mathrm{n}} \Rightarrow \widehat{\mathrm{Q}}(\widehat{\mathrm{m}}, t)=\widehat{\mathrm{Q}}(\widehat{\mathrm{n}}, t) \quad \forall \widehat{m}, \widehat{\mathrm{n}} \in \mathcal{H} .
$$

Accordingly, for indistinguishable $\widetilde{\mathcal{R}}$-particles, the representation of $\mathrm{Q}$ through

$$
\mathrm{Q}(\tilde{\mathrm{m}}, t):=\widehat{\mathrm{Q}}(\widehat{\mathrm{m}}, t)
$$

is well-defined.

The expectation value $\mathrm{Q}(\mathrm{M}, t)$ [or $\mathrm{Q}(\tilde{\mathrm{M}}, t)$, for indistinguishable $\widetilde{\mathcal{R}}$-particles] for the result of a measurement of $Q$ at time $t$ in the macrostate $M(\widetilde{M}$, respectively) is the weighted mean of the values that $\mathrm{Q}$ takes on at time $t$ in the individual microstates. Thus, for distinguishable $\mathcal{R}$-particles, it holds due to (5.32) that

$$
\mathrm{Q}(\mathrm{M}, t):=\int \mathrm{d} \mathcal{H} \rho_{\mathrm{M}}(\widehat{\mathrm{m}}) \widehat{\mathrm{Q}}(\widehat{\mathrm{m}}, t) .
$$

For indistinguishable $\widetilde{\mathcal{R}}$-particles, every microstate is represented by multiple phase space points such that, when calculating $\mathrm{Q}(\widetilde{\mathrm{M}}, t)$, one has to be careful to integrate over only one representative phase space point per microstate. According to (5.28), almost every equivalence class $[\widehat{\mathrm{m}}] \in \mathcal{H} / \sim$ possesses exactly one representative in $\widehat{\mu_{1,1}}$. Thus, for almost every microstate $\widetilde{\mathrm{m}}$ there is exactly one phase space point in $\widehat{\mu_{1,1}}$ representing $\widetilde{\mathrm{m}}$. Hence, it holds due to (5.35) that

$$
\mathrm{Q}(\widetilde{\mathrm{M}}, t):=\int_{\widehat{\mu_{1,1}}} \mathrm{~d} \mathcal{H} \rho_{\widetilde{\mathrm{M}}}(\widehat{\mathrm{m}}) \widehat{\mathrm{Q}}(\widehat{\mathrm{m}}, t)
$$

From this, considering (5.34), one obtains with a calculation analogous to (5.60),

$$
\mathrm{Q}(\widetilde{\mathrm{M}}, t)=\int \mathrm{d} \mathcal{H} \rho_{\mathrm{M}}(\widehat{\mathrm{m}}) \widehat{\mathrm{Q}}(\widehat{\mathrm{m}}, t) \stackrel{(5.36)}{=} \mathrm{Q}(\mathrm{M}, t) .
$$

In particular, for

$$
\widehat{\mathrm{Q}}(\widehat{\mathrm{m}}, t) \equiv 1
$$

one obtains the normalization condition for $\tilde{\mathrm{M}}$ :

$$
\begin{gathered}
\int_{\widehat{\mu_{1,1}}} \mathrm{~d} \mathcal{H} \rho_{\widetilde{\mathrm{M}}}(\widehat{\mathrm{m}}) \stackrel{(5.37),(5.39)}{=} \mathrm{Q}(\widetilde{\mathrm{M}}, t) \stackrel{(5.38)}{=} \mathrm{Q}(\mathrm{M}, t) \stackrel{(5.36),(5.39)}{=} \int \mathrm{d} \mathcal{H} \rho_{\mathrm{M}}(\widehat{\mathrm{m}}) \\
\stackrel{(2.10)}{=} 1 .
\end{gathered}
$$




\subsubsection{Temporal Evolution}

Let $\left\langle t_{0}\right\rangle:=\left\langle\zeta_{i} ; \vec{q}\left(t_{0}\right), \vec{p}\left(t_{0}\right)\right\rangle$ be a particular phase space point at time $t_{0}$ and, at any other point in time $t$, let $\langle t\rangle:=\left\langle\zeta_{i} ; \vec{q}(t), \vec{p}(t)\right\rangle$ be the phase space point unambiguously determined by the Hamilton equations

$$
\frac{\mathrm{d} q_{n}}{\mathrm{~d} t}=\frac{\partial \widehat{\mathrm{H}}}{\partial p_{n}}, \quad \frac{\mathrm{d} p_{n}}{\mathrm{~d} t}=-\frac{\partial \widehat{\mathrm{H}}}{\partial q_{n}} ; \quad n=1,2, \ldots, 3 N
$$

and the initial value $\left\langle t_{0}\right\rangle$. The Hamilton function $\widehat{\mathrm{H}}$ represents the mechanical quantity "energy" and is, according to (5.34), invariant under permutation of $\mathcal{R}$-particles. Thus, as will be shown below, if at time $t_{0}$ two phase space points $\left\langle t_{0}\right\rangle$ and $\left\langle t_{0}\right\rangle^{\prime}$ arise from each other through a certain permutation $\sigma$ of $\mathcal{R}$-particles, then, at any other time $t$, the phase space points $\langle t\rangle$ and $\langle t\rangle^{\prime}$ also arise from each other through $\sigma$ :

$$
\left\langle t_{0}\right\rangle^{\prime}=\sigma\left(\left\langle t_{0}\right\rangle\right) \quad \Leftrightarrow \quad\langle t\rangle^{\prime}=\sigma(\langle t\rangle)
$$

Proof The permutation $\sigma$ of $\mathcal{R}$-particles maps a phase space point $\left\langle\zeta_{i} ; \vec{q}, \vec{p}\right\rangle$ to the phase space point

$$
\begin{aligned}
\sigma\left(\left\langle\zeta_{i} ; \vec{q}, \vec{p}\right\rangle\right) & =\sigma\left(\left\langle\zeta_{i} ; q_{1}, \ldots, q_{3 N}, p_{1}, \ldots, p_{3 N}\right\rangle\right) \\
& =\left\langle\zeta_{j} ; q_{\pi(1)}, \ldots, q_{\pi(3 N)}, p_{\pi(1)}, \ldots, p_{\pi(3 N)}\right\rangle
\end{aligned}
$$

( $\pi$ is a particular element of the symmetric group of degree $3 N$.) Now, let it hold that

$$
\left\langle t_{0}\right\rangle^{\prime}=\sigma\left(\left\langle t_{0}\right\rangle\right)
$$

Then, between the coordinates of

$$
\left\langle t_{0}\right\rangle=\left\langle\zeta_{i} ; q_{1}\left(t_{0}\right), \ldots, p_{3 N}\left(t_{0}\right)\right\rangle \quad \text { and } \quad\left\langle t_{0}\right\rangle^{\prime}=\left\langle\zeta_{j} ; q_{1}^{\prime}\left(t_{0}\right), \ldots, p_{3 N}^{\prime}\left(t_{0}\right)\right\rangle
$$

there are, according to (5.43), the relations

$$
q_{n}^{\prime}\left(t_{0}\right)=q_{\pi(n)}\left(t_{0}\right) \quad \text { and } \quad p_{n}^{\prime}\left(t_{0}\right)=p_{\pi(n)}\left(t_{0}\right) ; \quad n=1,2, \ldots, 3 N .
$$

From this it follows that

$$
\begin{aligned}
& \frac{\partial \widehat{\mathrm{H}}\left(\left\langle t_{0}\right\rangle^{\prime}, t_{0}\right)}{\partial q_{n}^{\prime}} \stackrel{(5.45)}{=} \lim _{\Delta q \rightarrow 0} \frac{\widehat{\mathrm{H}}\left(\left\langle\zeta_{j} ; q_{1}^{\prime}\left(t_{0}\right), \ldots, q_{n}^{\prime}\left(t_{0}\right)+\Delta q, \ldots, p_{3 N}^{\prime}\left(t_{0}\right)\right\rangle, t_{0}\right)-\widehat{\mathrm{H}}\left(\left\langle t_{0}\right\rangle^{\prime}, t_{0}\right)}{\Delta q} \\
& \stackrel{(5.46)}{=} \lim _{\Delta q \rightarrow 0} \frac{\widehat{\mathrm{H}}\left(\langle\zeta_{j} ; q_{\pi(1)}\left(t_{0}\right), \ldots, \overbrace{q_{\pi(n)}\left(t_{0}\right)+\Delta q}^{n \text {th position }}, \ldots, p_{\pi(3 N)}\left(t_{0}\right)\rangle, t_{0}\right)-\widehat{\mathrm{H}}\left(\left\langle t_{0}\right\rangle^{\prime}, t_{0}\right)}{\Delta q} \\
& \stackrel{(5.43),(5.44)}{=} \lim _{\Delta q \rightarrow 0} \frac{\widehat{\mathrm{H}}\left(\sigma\left(\langle\zeta_{i} ; q_{1}\left(t_{0}\right), \ldots, \overbrace{q_{\pi(n)}\left(t_{0}\right)+\Delta q}^{\pi(n) \text { th position }}, \ldots, p_{3 N}\left(t_{0}\right)\rangle\right), t_{0}\right)-\widehat{\mathrm{H}}\left(\sigma\left(\left\langle t_{0}\right\rangle\right), t_{0}\right)}{\Delta q}
\end{aligned}
$$




$$
\stackrel{(5.34),(5.45)}{=} \frac{\partial \widehat{\mathrm{H}}\left(\left\langle t_{0}\right\rangle, t_{0}\right)}{\partial q_{\pi(n)}}
$$

and thus

$$
\begin{aligned}
p_{n}^{\prime}\left(t_{0}+\mathrm{d} t\right) & =p_{n}^{\prime}\left(t_{0}\right)+\left.\frac{\mathrm{d} p_{n}^{\prime}(t)}{\mathrm{d} t}\right|_{t=t_{0}} \mathrm{~d} t \stackrel{(5.41)}{=} p_{n}^{\prime}\left(t_{0}\right)-\frac{\partial \widehat{\mathrm{H}}\left(\left\langle t_{0}\right\rangle^{\prime}, t_{0}\right)}{\partial q_{n}^{\prime}} \mathrm{d} t \\
\stackrel{(5.46),(5.47)}{=} & p_{\pi(n)}\left(t_{0}\right)-\frac{\partial \widehat{\mathrm{H}}\left(\left\langle t_{0}\right\rangle, t_{0}\right)}{\partial q_{\pi(n)}} \mathrm{d} t \stackrel{(5.41)}{=} p_{\pi(n)}\left(t_{0}\right)+\left.\frac{\mathrm{d} p_{\pi(n)}(t)}{\mathrm{d} t}\right|_{t=t_{0}} \mathrm{~d} t \\
& =p_{\pi(n)}\left(t_{0}+\mathrm{d} t\right) .
\end{aligned}
$$

Correspondingly, it holds that

$$
q_{n}^{\prime}\left(t_{0}+\mathrm{d} t\right)=q_{\pi(n)}\left(t_{0}+\mathrm{d} t\right),
$$

and so, in total, one has

$$
\begin{aligned}
\left\langle t_{0}+\mathrm{d} t\right\rangle^{\prime} & =\left\langle\zeta_{j} ; q_{1}^{\prime}\left(t_{0}+\mathrm{d} t\right), \ldots, p_{3 N}^{\prime}\left(t_{0}+\mathrm{d} t\right)\right\rangle \\
\stackrel{(5.49),(5.48)}{=} & \left\langle\zeta_{j} ; q_{\pi(1)}\left(t_{0}+\mathrm{d} t\right), \ldots, p_{\pi(3 N)}\left(t_{0}+\mathrm{d} t\right)\right\rangle \\
\stackrel{(5.43)}{=} & \sigma\left(\left\langle\zeta_{i} ; q_{1}\left(t_{0}+\mathrm{d} t\right), \ldots, p_{3 N}\left(t_{0}+\mathrm{d} t\right)\right\rangle\right) \\
& =\sigma\left(\left\langle t_{0}+\mathrm{d} t\right\rangle\right) .
\end{aligned}
$$

Finally, if one divides the time period between $t_{0}$ and $t$ into an infinite number of infinitesimal time intervals, then the successive application of (5.50) on these intervals yields the claim (5.42).

If, at a certain point in time $t_{0}$, $\mathbb{S}$ is in the macrostate $\mathrm{M}\left(t_{0}\right)$ [or $\widetilde{\mathrm{M}}\left(t_{0}\right)$, for indistinguishable $\widetilde{\mathcal{R}}$-particles], then the macrostate $\mathrm{M}(t)[\widetilde{\mathrm{M}}(t)$, respectively] of $\mathbb{S}$ at time $t$ results from the temporal evolution of the phase space points; according to the Liouville equation, it holds that

$$
\rho_{\mathrm{M}(t)}(\langle t\rangle)=\rho_{\mathrm{M}\left(t_{0}\right)}\left(\left\langle t_{0}\right\rangle\right) \quad \forall\langle t\rangle \in \mathcal{H}
$$

and

$$
\rho_{\widetilde{\mathrm{M}}(t)}(\langle t\rangle)=\rho_{\widetilde{\mathrm{M}}\left(t_{0}\right)}\left(\left\langle t_{0}\right\rangle\right) \quad \forall\langle t\rangle \in \mathcal{H},
$$

respectively.

$\mathrm{M}(t)$ and $\tilde{\mathrm{M}}(t)$ are well-defined through (5.51) and (5.52): $\mathrm{M}(t)$ satisfies Postulate 5.1, because, if two phase space points $\langle t\rangle$ and $\langle t\rangle^{\prime}$ arise from each other through a permutation $\sigma$ of $\mathcal{R}$-particles according to

$$
\langle t\rangle^{\prime}=\sigma(\langle t\rangle),
$$

then it holds that

$$
\begin{aligned}
\rho_{\mathrm{M}(t)}\left(\langle t\rangle^{\prime}\right) & \stackrel{(5.51)}{=} \rho_{\mathrm{M}\left(t_{0}\right)}\left(\left\langle t_{0}\right\rangle^{\prime}\right) \stackrel{(5.53),(5.42)}{=} \rho_{\mathrm{M}\left(t_{0}\right)}\left(\sigma\left(\left\langle t_{0}\right\rangle\right)\right) \stackrel{\text { Postulate }}{=}{ }^{5.1} \rho_{\mathrm{M}\left(t_{0}\right)}\left(\left\langle t_{0}\right\rangle\right) \\
& \stackrel{(5.51)}{=} \rho_{\mathrm{M}(t)}(\langle t\rangle) .
\end{aligned}
$$


Further, $\mathrm{M}(t)$ satisfies the normalization condition (2.10) (the proof of this essentially proceeds as the proof in [11]). Analogously, it can be shown that $\widetilde{M}(t)$ satisfies the relation (5.31) and the normalization condition (5.40).

From

$$
\tilde{\mathrm{M}}\left(t_{0}\right):=\widetilde{\mathrm{M}\left(t_{0}\right)}
$$

at time $t_{0}$ it follows that for every other time $t$,

$$
\begin{aligned}
\rho_{\widetilde{\mathrm{M}}(t)}(\langle t\rangle) & \stackrel{(5.52)}{=} \rho_{\widetilde{\mathrm{M}}\left(t_{0}\right)}\left(\left\langle t_{0}\right\rangle\right) \stackrel{(5.55)}{=} \rho_{\widetilde{\mathrm{M}\left(t_{0}\right)}}\left(\left\langle t_{0}\right\rangle\right) \stackrel{(5.30)}{=}\left|\left[\left\langle t_{0}\right\rangle\right]\right| \rho_{\mathrm{M}\left(t_{0}\right)}\left(\left\langle t_{0}\right\rangle\right) \\
& \stackrel{(5.51)}{=}\left|\left[\left\langle t_{0}\right\rangle\right]\right| \rho_{\mathrm{M}(t)}(\langle t\rangle) \stackrel{(5.42)}{=}|[\langle t\rangle]| \rho_{\mathrm{M}(t)}(\langle t\rangle) \\
& \stackrel{(5.30)}{=} \rho_{\widetilde{\mathrm{M}(t)}}(\langle t\rangle) \quad \forall\langle t\rangle \in \mathcal{H}
\end{aligned}
$$

and thus

$$
\tilde{\mathrm{M}}(t)=\widetilde{\mathrm{M}(t)}
$$

\subsubsection{Mechanical Equivalence}

The expectation value for the result of a measurement of a mechanical quantity $Q$ is at any point in time $t$ the same for indistinguishable $\widetilde{\mathcal{R}}$-particles as for distinguishable $\mathcal{R}$-particles:

$$
\mathrm{Q}(\widetilde{\mathrm{M}}(t), t) \stackrel{(5.57)}{=} \mathrm{Q}(\widetilde{\mathrm{M}(t)}, t) \stackrel{(5.38)}{=} \mathrm{Q}(\mathrm{M}(t), t) .
$$

Thus, distinguishable $\mathcal{R}$-particles and indistinguishable $\widetilde{\mathcal{R}}$-particles are mechanically equivalent. In the next section (Sect. 5.6.6), it will be shown that distinguishable $\mathcal{R}$-particles and indistinguishable $\widetilde{\mathcal{R}}$-particles are equivalent with respect to the second law of thermodynamics as well. The equivalence with respect to the second law does not follow from the mechanical equivalence, since the entropy is not a mechanical quantity. Also, the second law is not a consequence of the Liouville equation, since the Liouville equation is entropy conserving [11] and therefore cannot describe irreversible processes (paradox of the constant entropy [48]).

\subsubsection{Entropy}

For indistinguishable $\widetilde{\mathcal{R}}$-particles, the entropy $S$ of a macrostate $\widetilde{M}$ is defined as

$$
S(\widetilde{\mathrm{M}}):=-k \int_{\widehat{\mu_{1,1}}} \mathrm{~d} \mathcal{H} \rho_{\widetilde{\mathrm{M}}}(\widehat{\mathrm{m}}) \ln \left[\rho_{\widetilde{\mathrm{M}}}(\widehat{\mathrm{m}})(2 \pi \hbar)^{3 N}\right] .
$$

[In the integration domain $\widehat{\mu_{1,1}}$, there is for almost every microstate $\tilde{\mathrm{m}}$ exactly one phase space point representing $\widetilde{\mathrm{m}}$ (cf. Sect. 5.6.3).]

5.6.6.1 Equivalence with Respect to the Second Law It holds that

$$
\begin{gathered}
S(\tilde{\mathrm{M}}) \underset{(5.30),(5.29)}{\stackrel{(5.59)}{=}}-k \int_{\widehat{\mu_{1,1}}} \mathrm{~d} \mathcal{H} \frac{\mathfrak{N} !}{(\mathfrak{N}-N) !} \rho_{\mathrm{M}}(\widehat{\mathrm{m}}) \ln \left[\frac{\mathfrak{N} !}{(\mathfrak{N}-N) !} \rho_{\mathrm{M}}(\widehat{\mathrm{m}})(2 \pi \hbar)^{3 N}\right] \\
\stackrel{\text { Postulate }}{=}{ }^{3.1}-k \sum_{i=1}^{\left(\begin{array}{c}
\mathfrak{N} \\
N
\end{array}\right)} \sum_{j=1}^{N !} \int_{\widehat{\mu_{1,1}}} \mathrm{~d} \mathcal{H} \rho_{\mathrm{M}}\left(\sigma_{i, j, 1,1}(\widehat{\mathrm{m}})\right)
\end{gathered}
$$




$$
\begin{aligned}
& \times \ln \left[\frac{\mathfrak{N} !}{(\mathfrak{N}-N) !} \rho_{\mathrm{M}}\left(\sigma_{i, j, 1,1}(\widehat{\mathrm{m}})\right)(2 \pi \hbar)^{3 N}\right] \\
\stackrel{(5.25)}{=} & -k \sum_{i=1}^{3} \sum_{j=1}^{N !} \int_{\widehat{\mu_{i, j}}} \mathrm{~d} \mathcal{H} \rho_{\mathrm{M}}(\widehat{\mathrm{m}}) \ln \left[\frac{\mathfrak{N} !}{(\mathfrak{N}-N) !} \rho_{\mathrm{M}}(\widehat{\mathrm{m}})(2 \pi \hbar)^{3 N}\right] \\
\stackrel{(5.27),(5.24)}{=} & -k \int \mathrm{d} \mathcal{H} \rho_{\mathrm{M}}(\widehat{\mathrm{m}}) \ln \left[\frac{\mathfrak{N} !}{(\mathfrak{N}-N) !} \rho_{\mathrm{M}}(\widehat{\mathrm{m}})(2 \pi \hbar)^{3 N}\right] \\
\stackrel{(2.10)}{=} & -k \int \mathrm{d} \mathcal{H} \rho_{\mathrm{M}}(\widehat{\mathrm{m}}) \ln \left[\rho_{\mathrm{M}}(\widehat{\mathrm{m}})(2 \pi \hbar)^{3 N}\right]-k \ln \frac{\mathfrak{N} !}{(\mathfrak{N}-N) !} \\
\stackrel{(2.11)}{=} & S(\mathrm{M})-k \ln \frac{\mathfrak{N} !}{(\mathfrak{N}-N) !} .
\end{aligned}
$$

Since $\mathbb{S}$ is closed with respect to particle exchange and, according to Sect. $2.3, \mathcal{R}$-particles can neither be created nor annihilated during the observation period, $k \ln \frac{\mathfrak{N} !}{(\mathfrak{N}-N) !}$ is constant in time. Thus, the change in entropy that $\mathbb{S}$ experiences during an examined process is the same for distinguishable $\mathcal{R}$-particles as for indistinguishable $\widetilde{\mathcal{R}}$-particles. Hence, distinguishable $\mathcal{R}$-particles and indistinguishable $\widetilde{\mathcal{R}}$-particles are equivalent with respect to the second law of thermodynamics. In particular, the GP1 does not pose a real contradiction to the second law for either distinguishable $\mathcal{R}$-particles or for indistinguishable $\widetilde{\mathcal{R}}$-particles (see Sects. 6.1.2 and 3.1.3).

5.6.6.2 Thermodynamic Potential As mentioned in Sect. 4.3.3.2, for indistinguishable $\widetilde{\mathcal{R}}$ particles, the equilibrium entropy as a function of its natural variables $E, N, \ldots$ is a thermodynamic potential; for distinguishable $\mathcal{R}$-particles this role is adopted by the reduced entropy (see Sect. 5.5). Due to

$$
R(\mathrm{M}) \stackrel{(4.39)}{=} S(\mathrm{M})-k \ln \frac{\mathfrak{N} !}{(\mathfrak{N}-N) !} \stackrel{(5.60)}{=} S(\tilde{\mathrm{M}}),
$$

the reduced entropy for distinguishable $\mathcal{R}$-particles agrees with the entropy for indistinguishable $\widetilde{\mathcal{R}}$-particles, and so the thermodynamic potentials are the same in both cases. This confirms the equivalence with respect to the second law between distinguishable $\mathcal{R}$-particles and indistinguishable $\widetilde{\mathcal{R}}$-particles.

Remark 5.5 From (5.61) and (4.41) it follows that systems consisting of distinguishable $\mathcal{R}$ particles that are correlated only by Condition 4.5 become uncorrelated in the course of the transition to indistinguishable $\widetilde{\mathcal{R}}$-particles.

5.6.6.3 Limiting Case $\mathfrak{N} \gg N$ (Inherent Entropy) If the number $\mathfrak{N}$ of particles in the underlying particle set $\mathcal{R}$ is very much larger than the number $N$ of the $\mathcal{R}$-particles in $\mathbb{S}$, then the difference between $S(\mathrm{M})$ and $S(\widetilde{\mathrm{M}})$ can be simplified:

$$
S(\mathrm{M})-S(\tilde{\mathrm{M}}) \stackrel{(5.60)}{=} k \ln \frac{\mathfrak{N} !}{(\mathfrak{N}-N) !} \stackrel{\mathfrak{N} \gg N}{\approx} k \ln \mathfrak{N}^{N}=N k \ln \mathfrak{N} .
$$

Thus, for distinguishable $\mathcal{R}$-particles, in the case $\mathfrak{N} \gg N$, every $\mathcal{R}$-particle possesses an additional inherent entropy (i.e., an additional entropy intrinsically tied to the particle) of $k \ln \mathfrak{N}$ compared to indistinguishable $\widetilde{\mathcal{R}}$-particles. An $\mathcal{R}$-particle brings with it this entropy 
when it is added to $\mathbb{S}$ and it carries this entropy away when it is removed from $\mathbb{S}$. The inherent entropy of an $\mathcal{R}$-particle reflects the uncertainty about its identity: it is in principle not possible to determine which of the $\mathfrak{N} \mathcal{R}$-particles the particle in question is.

Remark 5.6 If the $N \mathcal{R}$-particles form an ideal gas, then the entropy $S(T, N, V)$ of this gas becomes extensive in the limit $\mathfrak{N} \gg N$ :

$$
\begin{aligned}
S(T, N, V) & \stackrel{(5.62)}{=} \widetilde{S}(T, N, V)+N k \ln \mathfrak{N} \\
& \stackrel{(3.7)}{=} N k\left[\ln \frac{V}{N}+\frac{3}{2} \ln \frac{m k T}{2 \pi \hbar^{2}}+\frac{5}{2}+\ln \mathfrak{N}\right] .
\end{aligned}
$$

Together with the additivity of the entropy for $\mathfrak{N} \gg N$ [cf. (5.18) and Remark 4.8], this leads to the disappearance of the GP1 in the limit $\mathfrak{N} \gg N$. The absence of the GP1 in the limiting case $\mathfrak{N} \gg N$ was found before by van Kampen [44].

\section{Resolution of the GP1}

\subsection{Distinguishable Identical Classical Particles}

Let a set $\mathcal{R}$ of $\mathfrak{N}$ pairwise distinguishable identical classical particles be given. Let the $\mathcal{R}$ particles each have mass $m$ and no internal degrees of freedom.

\subsubsection{Entropy of an Ideal Gas}

As in Sect. 3.1.1, for an ideal gas of $N(N \gg 1) \mathcal{R}$-particles confined to a vessel of volume $V$ at temperature $T$, (3.4) can be derived for the entropy. However, in the derivation of (3.4) the possibility that there are particles of the same kind outside the vessel was disregarded; thus, in the case of an ideal gas of $N \mathcal{R}$-particles, (3.4) is not the entropy of the equilibrium state characterized by $T, N$, and $V$, but, according to Theorem 5.3, the entropy of the minimal state associated with that equilibrium state:

$$
S\left(\underline{T, N, V)}=N k\left[\ln V+\frac{3}{2} \ln \frac{m k T}{2 \pi \hbar^{2}}+\frac{3}{2}\right] .\right.
$$

As per (5.12), this yields the entropy

$$
S(T, N, V)=N k\left[\ln V+\frac{3}{2} \ln \frac{m k T}{2 \pi \hbar^{2}}+\frac{3}{2}\right]+k \ln \left(\begin{array}{c}
\mathfrak{N} \\
N
\end{array}\right)
$$

for the equilibrium state characterized by $T, N$, and $V$.

\subsubsection{Resolution of the GP1}

Two vessels of equal volume $V$ each containing an ideal gas of $N \mathcal{R}$-particles are combined such that a single vessel of total volume $2 \mathrm{~V}$ containing an ideal gas of $2 N \mathcal{R}$-particles is formed. The entropy of this combined system is $S(T, 2 N, 2 V)$. Prior to the combination, the two subsystems (i.e., the two individual vessels) each had an entropy of $S(T, N, V)$.

Since the function $S(T, N, V)$ in (6.2) is not homogeneous of degree 1 in the variables $N$ and $V$, it generally holds that $S(T, 2 N, 2 V) \neq 2 S(T, N, V)$. Thus, if the entropy of the 
initial system consisting of the two individual vessels would be the sum of the entropies of its two subsystems, that is, $2 S(T, N, V)$, then the process of combining paradoxically would be associated with a change in entropy (see Sect. 3.1.1). This, however, is not the case; according to (5.18), the entropy of the initial system is

$$
\begin{aligned}
S(T, N, V)+ & S(T, N, V)-k \ln \frac{\mathfrak{N} !(\mathfrak{N}-2 N) !}{(\mathfrak{N}-N) !(\mathfrak{N}-N) !} \\
& \stackrel{(6.2)}{=} 2 N k\left[\ln V+\frac{3}{2} \ln \frac{m k T}{2 \pi \hbar^{2}}+\frac{3}{2}\right]+k \ln \left[\frac{\mathfrak{N} !}{(\mathfrak{N}-2 N) ! N ! N !}\right] \\
& \stackrel{(6.4)}{\approx} 2 N k\left[\ln 2 V+\frac{3}{2} \ln \frac{m k T}{2 \pi \hbar^{2}}+\frac{3}{2}\right]+k \ln \left(\begin{array}{c}
\mathfrak{N} \\
2 N
\end{array}\right) \\
& \stackrel{(6.2)}{=} S(T, 2 N, 2 V)
\end{aligned}
$$

and thus is equal to the entropy of the system after the combination. Hence, the process of combining does not lead to a change in entropy; the GP1 is resolved.

Remark 6.1 In (6.3), the relation

$$
k \ln \left[\frac{\mathfrak{N} !}{(\mathfrak{N}-2 N) ! N ! N !}\right]=k \ln \left[\left(\begin{array}{c}
\mathfrak{N} \\
2 N
\end{array}\right)\left(\begin{array}{c}
2 N \\
N
\end{array}\right)\right] \stackrel{(3.11)}{\approx} k \ln \left(\begin{array}{c}
\mathfrak{N} \\
2 N
\end{array}\right)+2 N k \ln 2,
$$

which holds only approximately, was applied. An exact calculation yields a slightly larger entropy for the combined system than for the initial system because after the combination there need no longer be exactly $N$ particles in each of the two subsystems [5].

Remark 6.2 If, for an ideal gas of $N$ distinguishable identical classical particles confined to a vessel of volume $V$ at temperature $T$, the "localization within the vessel" is declared as an inner attribute (cf. the second example in Sect. 2.6.2), then there is no uncertainty about which particles are located in the vessel and (3.4) correctly expresses the entropy of the ideal gas (cf. Sect. 6.1.1). However, if the "localization within the vessel" is declared as an inner attribute, a combination of the vessel with another is ruled out (see Sect. 2.4), and so in this case the GP1 does not arise in the first place.

Remark 6.3 Using the reduced entropy, the resolution of the GP1 can be shortened as follows. For the reduced entropy of an ideal gas of $N \mathcal{R}$-particles, the Sackur-Tetrode equation holds:

$$
\begin{gathered}
R(T, N, V) \stackrel{(5.19),(6.1)}{=} N k\left[\ln V+\frac{3}{2} \ln \frac{m k T}{2 \pi \hbar^{2}}+\frac{3}{2}\right]-k \ln N ! \\
\stackrel{\text { Stirling }}{\approx} N k\left[\ln \frac{V}{N}+\frac{3}{2} \ln \frac{m k T}{2 \pi \hbar^{2}}+\frac{5}{2}\right] .
\end{gathered}
$$

The Sackur-Tetrode equation is homogeneous of degree 1 in the variables $N$ and $V$. This, together with the additivity (4.41) of the reduced entropy, implies that, in the case of the two vessels considered above, the reduced entropy of the initial system equals the reduced entropy of the combined system. Thus, due to (4.42), the entropies of the initial and the combined system agree as well. 


\subsection{Non-identical Particles}

Let a set $\mathcal{P}:=\left\{\mathfrak{p}_{1}, \mathfrak{p}_{2}, \ldots, \mathfrak{p}_{\mathfrak{N}}\right\}$ of $\mathfrak{N}$ pairwise non-identical (quantum or classical) particles be given. For simplicity, let the $\mathcal{P}$-particles have no internal degrees of freedom; let $m_{l}$ be the mass of the $\mathcal{P}$-particle $\mathfrak{p}_{l}(l=1,2, \ldots, \mathfrak{N})$.

\subsubsection{Entropy of a $\mathcal{P}$-harmonic Ideal Gas}

Consider an ideal gas $\mathbb{S}$ of $N \mathcal{P}$-particles confined to a vessel of volume $V$ at temperature $T$. There are $\left(\begin{array}{c}\mathfrak{N} \\ N\end{array}\right)$ compositions $\zeta_{1}, \zeta_{2}, \ldots, \zeta_{\left(\begin{array}{l}\mathfrak{N} \\ N\end{array}\right)}$ of $\mathbb{S}$ that consist of $N \mathcal{P}$-particles. Let the composition $\zeta_{i}$ contain the $\mathcal{P}$-particles $\mathfrak{p}_{n_{i, 1}}, \mathfrak{p}_{n_{i, 2}}, \ldots, \mathfrak{p}_{n_{i, N}}\left(n_{i, 1}, n_{i, 2}, \ldots, n_{i, N} \in\{1,2, \ldots, \mathfrak{N}\}\right)$.

If $\mathbb{S}$ has the composition $\zeta_{i}$ with certainty, then (3.1) yields its canonical partition function

$$
Z\left(T, \zeta_{i}, V\right)=\prod_{j=1}^{N} V\left(\frac{m_{n_{i, j}} k T}{2 \pi \hbar^{2}}\right)^{3 / 2}=\left[V\left(\frac{\bar{m}_{i} k T}{2 \pi \hbar^{2}}\right)^{3 / 2}\right]^{N}
$$

where

$$
\bar{m}_{i}:=\sqrt[N]{\prod_{j=1}^{N} m_{n_{i, j}}}
$$

is the geometric mean of the masses of all $\zeta_{i}$-particles. From $Z\left(T, \zeta_{i}, V\right)$ one obtains the entropy

$$
S\left(T, \zeta_{i}, V\right) \stackrel{(3.3)}{=} N k\left[\ln V+\frac{3}{2} \ln \frac{\bar{m}_{i} k T}{2 \pi \hbar^{2}}+\frac{3}{2}\right] .
$$

Now, let $\mathbb{S}$ be $\mathcal{P}$-harmonic. According to Theorem 4.4, it holds for the conditional entropy with respect to $\zeta_{i}$ that

$$
S\left(T, V \mid \zeta_{i}\right)=S\left(T, \zeta_{i}, V\right) .
$$

With this, the arithmetic mean of all conditional entropies of $\mathbb{S}$ is

$$
\bar{S}(T, N, V)=N k\left[\ln V+\frac{3}{2} \ln \frac{\bar{m} k T}{2 \pi \hbar^{2}}+\frac{3}{2}\right],
$$

where

$$
\bar{m}:=\sqrt[\mathfrak{N}]{\prod_{l=1}^{\mathfrak{N}} m_{l}}
$$

is the geometric mean of the masses of all $\mathcal{P}$-particles.

Proof It holds that

$$
\begin{aligned}
\bar{S}(T, N, V) & \stackrel{(6.9)}{=} \frac{1}{\left(\begin{array}{l}
\mathfrak{N} \\
N
\end{array}\right)} \sum_{i=1}^{\left(\begin{array}{c}
\mathfrak{N} \\
N
\end{array}\right)} S\left(T, \zeta_{i}, V\right) \\
\stackrel{(6.8),(6.7)}{=} & N k\left[\ln V+\frac{3}{2} \ln \frac{k T}{2 \pi \hbar^{2}}+\frac{3}{2}+\frac{3}{2} \frac{1}{\left(\begin{array}{l}
\mathfrak{N} \\
N
\end{array}\right)} \frac{1}{N} \sum_{i=1}^{\left(\begin{array}{l}
\mathfrak{N} \\
N
\end{array}\right)} \sum_{j=1}^{N} \ln m_{n_{i, j}}\right] .
\end{aligned}
$$


Each of the $\mathfrak{N} \mathcal{P}$-particles occurs in a total of $\left(\begin{array}{l}\mathfrak{N}-1 \\ N-1\end{array}\right)$ of the $\left(\begin{array}{l}\mathfrak{N} \\ N\end{array}\right)$ compositions $\left.\zeta_{1}, \zeta_{2}, \ldots, \zeta_{(}^{\mathfrak{N}}\right)$; hence, the double sum in (6.12) can be rearranged as follows:

$$
\sum_{i=1}^{\left(\begin{array}{l}
\mathfrak{N} \\
N
\end{array}\right)} \sum_{j=1}^{N} \ln m_{n_{i, j}}=\left(\begin{array}{l}
\mathfrak{N}-1 \\
N-1
\end{array}\right) \sum_{l=1}^{\mathfrak{N}} \ln m_{l} .
$$

One then obtains

$$
\begin{gathered}
\bar{S}(T, N, V) \stackrel{(6.12),(6.13)}{=} N k\left[\ln V+\frac{3}{2} \ln \frac{k T}{2 \pi \hbar^{2}}+\frac{3}{2}+\frac{3}{2} \frac{1}{\mathfrak{N}} \sum_{l=1}^{\mathfrak{N}} \ln m_{l}\right] \\
\stackrel{(6.11)}{=} N k\left[\ln V+\frac{3}{2} \ln \frac{\bar{m} k T}{2 \pi \hbar^{2}}+\frac{3}{2}\right] .
\end{gathered}
$$

Finally, plugging (6.10) into (4.23) yields the entropy of $\mathbb{S}$ :

$$
S(T, N, V)=N k\left[\ln V+\frac{3}{2} \ln \frac{\bar{m} k T}{2 \pi \hbar^{2}}+\frac{3}{2}\right]+k \ln \left(\begin{array}{c}
\mathfrak{N} \\
N
\end{array}\right) .
$$

\subsubsection{Resolution of the GP1 for P-harmonic Ideal Gases}

Now, consider an ideal gas $\mathbb{S}$ consisting, for simplicity, of all $\mathfrak{N}(\mathfrak{N} \gg 1) \mathcal{P}$-particles, which is confined to a vessel of volume $V$ at temperature $T$. Trivially, $\mathbb{S}$ is $\mathcal{P}$-harmonic since there is only one possible composition.

$\mathbb{S}$ is then divided into two equal subsystems $\mathbb{S}_{1}$ and $\mathbb{S}_{2}\left(N_{1}=N_{2}=\frac{\mathfrak{N}}{2}, V_{1}=V_{2}=\frac{V}{2}\right)$ by inserting a partition. $\mathbb{S}_{1}$ and $\mathbb{S}_{2}$ are $\mathcal{P}$-harmonic as well, since all $\left(\begin{array}{c}\mathfrak{N} \\ \frac{\mathfrak{N}}{2}\end{array}\right)$ possibilities of how the $\mathfrak{N} \mathcal{P}$-particles can be distributed over the two subsystems are equiprobable.

$\mathbb{S}_{1}$ and $\mathbb{S}_{2}$ can be recombined to obtain the original system $\mathbb{S}$ by removing the partition. As reasoned in Sect. 3.1.1, a change in entropy in the course of this recombination would be paradoxical (GP1). However, such an entropy change does not occur: $\mathbb{S}_{1}, \mathbb{S}_{2}$, and $\mathbb{S}$ are all $\mathcal{P}$-harmonic ideal gases. Comparison of (6.14) with (6.2) shows that the entropy of a $\mathcal{P}$-harmonic ideal gas agrees with the entropy of an ideal gas of distinguishable identical classical particles of mass $\bar{m}$. Thus, applying (4.38), the GP1 can be resolved as in Sect. 6.1.2.

\subsubsection{Ideal Gases that Consist of $\mathcal{P}$-particles and Are Not $\mathcal{P}$-harmonic}

Now, let the ideal gas $\mathbb{S}$ of all $\mathfrak{N} \mathcal{P}$-particles again be partitioned into two equal subsystems $\mathbb{S}_{3}$ and $\mathbb{S}_{4}\left(N_{3}=N_{4}=\frac{\mathfrak{N}}{2}, V_{3}=V_{4}=\frac{V}{2}\right)$. However, this time, let $\mathbb{S}_{3}$ and $\mathbb{S}_{4}$ not be $\mathcal{P}$ harmonic.

In order to prepare $\mathbb{S}_{3}$ and $\mathbb{S}_{4}$ such that they are not $\mathcal{P}$-harmonic, one may, for example, proceed as follows: One exposes the $\mathfrak{N} \mathcal{P}$-particles of $\mathbb{S}$ to a vertical gravitational field and then inserts a horizontal partition into $\mathbb{S}$. The position at which the partition is inserted is chosen such that the upper and the lower subsystem each contain $\frac{\mathfrak{N}}{2} \mathcal{P}$-particles. Due to the gravitation, in the lower subsystem, compositions of heavier $\mathcal{P}$-particles have a higher probability than compositions of lighter $\mathcal{P}$-particles; in the upper subsystem, the opposite is 
the case. Hence, neither subsystem is $\mathcal{P}$-harmonic. After the partitioning of $\mathbb{S}$, one continues by adjusting the volumes of the two subsystems to $\frac{V}{2}$ by moving the partition. In doing so, a particle exchange between the subsystems must be prevented in order for the composition probabilities to remain unaltered.

By removing the partition, $\mathbb{S}_{3}$ and $\mathbb{S}_{4}$ can be recombined to a single undivided system of total volume $V$ and particle number $\mathfrak{N}$. In the absence of a gravitational field this recombination is an irreversible process, since, if one reinserts the partition after equilibrium is reached, then one obtains, instead of $\mathbb{S}_{3}$ and $\mathbb{S}_{4}$, two $\mathcal{P}$-harmonic systems (see Sect. 6.2.2). Hence, the process of combining $\mathbb{S}_{3}$ and $\mathbb{S}_{4}$ causes an increase in entropy, which of course is not paradoxical.

Remark 6.4 Of course, the process of combining two ideal gases that consist of $\mathcal{P}$-particles and are not $\mathcal{P}$-harmonic is not necessarily associated with an entropy increase. If, for example, in equilibrium a partition is inserted into an ideal gas that consists of $\mathcal{P}$-particles and is not $\mathcal{P}$-harmonic, then one obtains two ideal gases that also consist of $\mathcal{P}$-particles and are not $\mathcal{P}$-harmonic. By removing the partition, these two gases can be recombined to the original gas without changing the entropy.

\section{Conclusion}

The analysis of the GP1 in Sect. 3.2 shows that, for systems of distinguishable particles, it is generally uncertain of which particles they consist. In order to be able to describe an ensemble of possible particle compositions, the quantum-mechanical state space was defined in Sect. 2.1.1 as the direct sum of many-particle state spaces (each many-particle state space corresponding to a certain particle composition); analogously, the union of multiple phase spaces was introduced in Sect. 2.1.2 as the set of representatives for classical microstates. Further, in Sect. 2.2, the conventional definition for particle transposition was extended to the case of the transposition of a system particle with a particle that is not a system particle. The statistical treatment of systems of distinguishable particles was discussed in Sects. 4 (non-identical particles) and 5 (distinguishable identical classical particles). In both cases, an underlying particle set, containing all particles that in principle qualify for being part of the regarded system, was assumed to be known. First, in Sect. 4.1, it was shown that, for systems of distinguishable particles, the uncertainty about the particle composition contributes to the entropy. Systems of distinguishable particles for which all possible compositions are equiprobable were referred to as harmonic (Sect. 4.3). Whereas systems of distinguishable identical classical particles are always harmonic due to Postulate 5.1 (cf. Sect. 5.2), harmonicity is not a necessary property for systems of non-identical particles. Since in addition the probabilities of the possible compositions of a closed system cannot change over the course of time, for closed systems of non-identical particles the composition probabilities are part of the complete characterization of an equilibrium state (Sect. 4.2.1). In Sect. 4.3.2, it was noted that systems that are harmonic with respect to the same underlying particle set are always correlated; thus, for harmonic systems, the entropy is not additive and, as a consequence, loses its thermodynamic meaning. For harmonic systems, the reduced entropy (instead of the entropy) is a thermodynamic potential (Sects. 4.3.3 and 5.5). In Sect. 5.6, it was demonstrated that one has the choice of assuming identical classical particles to be distinguishable or indistinguishable; both possibilities are mechanically equivalent (Sect. 5.6.5) as well as equivalent with respect to the second law of thermodynamics (Sect. 5.6.6.1). Finally, in Sect. 6, the resolution of the GP1 was demonstrated, applying the results deduced 
in Sects. 4 and 5 (in Sect. 6.1.2 for gases of distinguishable identical classical particles, and in Sect. 6.2.2 for harmonic gases of non-identical particles).

The resolution of the GP1 for distinguishable identical classical particles (Sect. 6.1.2) closes a gap in the theory of classical statistical mechanics; contrary to popular belief $[19,21,38]$, identical classical particles can be assumed to be distinguishable without contradiction. According to Sect. 5.6, it is not a matter of right or wrong whether one assumes identical classical particles to be distinguishable or indistinguishable, but a matter of taste: An argument for distinguishability is that one can adhere to the intuitive notion of particles as individual objects; further, one deals with simpler integration domains if one assumes identical classical particles to be distinguishable [17]. An argument for indistinguishability is Ockham's razor, since in this case there are no microstates that are experimentally indiscernible; a further advantage of indistinguishable classical particles is that the entropy generally is extensive and additive.

As already emphasized in Sect. 1, the GP1 does not only affect classical statistical mechanics; quantum systems can suffer from the GP1 as well (Sect. 3.1.1). Hence, the GP1 wrongfully faded into the background after the discovery of quantum mechanics.

The statistics of non-identical particles, presented in Sect. 4, does not only carry theoretical weight; it is also fundamental for the statistical description of colloids (such as homogenized milk [41]). The equivalence between distinguishable and indistinguishable identical classical particles, proven in Sect. 5.6, is, among other things, interesting from a philosophical view (see, e.g., $[33,36]$ and references therein). Last but not least, the results of the present article shed new light on the GP2. The reason for the point of discontinuity, which the GP2 addresses (cf. Sect. 1), depends on whether the particles remain distinguishable or become indistinguishable at the transition from 'similar' to 'identical'. If the particles remain distinguishable, then, in the 'identical' limiting case (and only then), Postulate 5.1 takes effect and forces the harmonicity of the two gases with respect to the same underlying particle set.

Open Access This article is distributed under the terms of the Creative Commons Attribution Noncommercial License which permits any noncommercial use, distribution, and reproduction in any medium, provided the original author(s) and source are credited.

\section{References}

1. Baierlein, R.: The fraction of "all different" combinations: Justifying the semi-classical partition function. Am. J. Phys. 65, 314-316 (1997)

2. Ben-Naim, A.: On the so-called Gibbs paradox, and on the real paradox. Entropy 9, 132-136 (2007)

3. Boyer, T.H.: Sharpening Bridgman's resolution of the Gibbs paradox. Am. J. Phys. 38, 771-773 (1970)

4. Bridgman, P.W.: The Nature of Thermodynamics, pp. 168-169. Harper, New York (1961)

5. Casper, B.M., Freier, S.: "Gibbs paradox" paradox. Am. J. Phys. 41, 509-511 (1973)

6. Cheng, C.H.: Thermodynamics of the system of distinguishable particles. Entropy 11, 326-333 (2009)

7. Denbigh, K.G., Redhead, M.L.G.: Gibbs' paradox and non-uniform convergence. Synthese 81, 283-312 (1989)

8. Diu, B., Guthmann, C., Lederer, D., Roulet, B.: Grundlagen der Statistischen Physik, p. 170. de Gruyter, Berlin (1994)

9. Diu, B., Guthmann, C., Lederer, D., Roulet, B.: Grundlagen der Statistischen Physik, p. 375. de Gruyter, Berlin (1994)

10. Diu, B., Guthmann, C., Lederer, D., Roulet, B.: Grundlagen der Statistischen Physik, pp. 762-763, 804805. de Gruyter, Berlin (1994)

11. Ehrenfest, P., Ehrenfest, T.: The Conceptual Foundations of the Statistical Approach in Mechanics, p. 54, 98. Cornell University Press, Ithaca (1959)

12. Ehrenfest, P., Oppenheimer, J.R.: Note on the statistics of nuclei. Phys. Rev. 37, 333-338 (1931) 
13. Ehrenfest, P., Trkal, V.: Ableitung des Dissoziationsgleichgewichtes aus der Quantentheorie und darauf beruhende Berechnung der chemischen Konstanten. Ann. Phys. (Leipzig) 65(370), 609-628 (1921). Remark of Ehrenfest on p. 628

14. Einstein, A., Podolsky, B., Rosen, N.: Can quantum-mechanical description of physical reality be considered complete? Phys. Rev. 47, 777-780 (1935)

15. Gemmer, J., Michel, M., Mahler, G.: Quantum Thermodynamics, 2 edn., pp. 129-131. Springer, Berlin (2009)

16. Gibbs, J.W.: Elementary Principles in Statistical Mechanics, pp. 206-207. Scribner's, New York (1902). Reprinted by Dover, New York (1960)

17. Gibbs, J.W.: Elementary Principles in Statistical Mechanics, p. 188. Scribner's, New York (1902). Reprinted by Dover, New York (1960)

18. Gibbs, J.W.: On the equilibrium of heterogeneous substances. In: The Scientific Papers of J. Willard Gibbs, vol. 1, pp. 165-168. Dover, New York (1961). First published in Transactions of the Connecticut Academy of Arts and Sciences, vol. 3, New Haven (1875-1878)

19. Hestenes, D.: Entropy and indistinguishability. Am. J. Phys. 38, 840-845 (1970)

20. Home, D., Sengupta, S.: Extensivity of entropy and modern form of Gibbs paradox. Pramana 17, 509514 (1981)

21. Huang, K.: Statistical Mechanics, pp. 153-155. Wiley, New York (1963)

22. Jaynes, E.T.: The Gibbs paradox. In: Smith, C.R. et al. (ed.) Maximum Entropy and Bayesian Methods, Seattle, 1991, pp. 1-21. Kluwer, Dordrecht (1992)

23. Landé, A.: New Foundations of Quantum Mechanics, pp. 64-66. Cambridge University Press, Cambridge (1965)

24. Landé, A.: New Foundations of Quantum Mechanics, pp. 61-64. Cambridge University Press, Cambridge (1965)

25. Lyuboshitz, V.L., Podgoretskii, M.I.: The Gibbs paradox. Sov. Phys. Usp. 14, 662-666 (1972)

26. Messiah, A.: Quantum Mechanics, vol. 2, pp. 619-626. North-Holland, Amsterdam (1962)

27. Messiah, A.: Quantum Mechanics, vol. 2, pp. 600-603. North-Holland, Amsterdam (1962)

28. Messiah, A.M.L., Greenberg, O.W.: Symmetrization postulate and its experimental foundation. Phys. Rev. 136, B248-B267 (1964)

29. Nagle, J.F.: Regarding the entropy of distinguishable particles. J. Stat. Phys. 117, 1047-1062 (2004)

30. Pathria, R.K.: Statistical Mechanics, 2 edn., pp. 22-26, 122. Butterworth-Heinemann, Oxford (1996)

31. Planck, M.: Vorlesungen über die Theorie der Wärmestrahlung, 4th edn., pp. 209-210. Barth, Leipzig (1921)

32. Planck, M.: Absolute Entropie und chemische Konstante. Ann. Phys. (Leipzig) 66(371), 365-372 (1921). Reprinted in Planck, M.: Physikalische Abhandlungen und Vorträge, vol. 2, pp. 527-534. Vieweg, Braunschweig (1958)

33. Pniower, J.C.: Particles, objects, and physics. Ph.D. thesis. Oxford University, Oxford (2005)

34. Rushbrooke, G.S.: Introduction to Statistical Mechanics, pp. 36-39. Clarendon, Oxford (1949)

35. Rushbrooke, G.S.: Introduction to Statistical Mechanics, pp. 40-44, 61-63. Clarendon, Oxford (1949)

36. Saunders, S.: On the explanation for quantum statistics. Stud. Hist. Philos. Mod. Phys. 37, 192-211 (2006)

37. Schrödinger, E.: Gasentartung und freie Weglänge. Phys. Z. 25, 41-45 (1924). Reprinted in Schrödinger, E.: Gesammelte Abhandlungen, vol. 1, pp. 319-323. Österreichische Akademie der Wissenschaften, Wien (1984)

38. Schrödinger, E.: Statistical Thermodynamics, pp. 58-62. Cambridge University Press, Cambridge (1948)

39. Swendsen, R.H.: Statistical mechanics of classical systems with distinguishable particles. J. Stat. Phys. 107, 1143-1166 (2002)

40. Swendsen, R.H.: Response to Nagle's criticism of my proposed definition of the entropy. J. Stat. Phys. 117, 1063-1070 (2004)

41. Swendsen, R.H.: Statistical mechanics of colloids and Boltzmann's definition of the entropy. Am. J. Phys. 74, 187-190 (2006)

42. ter Haar, D.: Elements of Statistical Mechanics, pp. 71-72. Rinehart, New York (1954)

43. Tetrode, H.: Die chemische Konstante der Gase und das elementare Wirkungsquantum. Ann. Phys. (Leipzig) 38(343), 434-442 (1912)

44. van Kampen, N.G.: The Gibbs paradox. In: Parry, W.E. (ed.) Essays in Theoretical Physics in Honour of Dirk ter Haar, pp. 303-312. Pergamon, Oxford (1984) 
45. van Kampen, N.G.: The Gibbs paradox. In: Parry, W.E. (ed.) Essays in Theoretical Physics in Honour of Dirk ter Haar, p. 308. Pergamon, Oxford (1984)

46. von Neumann, J.: Mathematical Foundations of Quantum Mechanics, pp. 370-376. Princeton University Press, Princeton (1955)

47. Warren, P.B.: Combinatorial entropy and the statistical mechanics of polydispersity. Phys. Rev. Lett. 80, 1369-1372 (1998)

48. Wehrl, A.: General properties of entropy. Rev. Mod. Phys. 50, 221-260 (1978) 